\title{
Phosphorylation of LCB1 subunit of serine palmitoyltransferase stimulates its activity and modulates sphingolipid biosynthesis in Arabidopsis
}

\section{Yuan Li}

State Key Laboratory of Plant Physiology and Biochemistry, College of Biological Sciences, China Agricultural University

\section{Hanwei Cao}

State Key Laboratory of Plant Physiology and Biochemistry, College of Biological Sciences, China Agricultural University

\section{Tingting Dong}

State Key Laboratory of Plant Physiology and Biochemistry, College of Biological Sciences, China Agricultural University

\section{Xiaoke Wang}

State Key Laboratory of Plant Physiology and Biochemistry, College of Biological Sciences, China Agricultural University

\section{Liang Ma}

China Agricultural University

Kun Li

Collaborative Innovation Center of Crop Stress Biology, Henan Province. Institute of Plant Stress Biology, School of Life Science, Henan University

\section{Huqiang Lou}

China Agricultural University https://orcid.org/0000-0003-4465-6186

\section{Chun-Peng Song}

Henan University https://orcid.org/0000-0001-8774-4309

Dongtao Ren ( $\nabla$ ren@cau.edu.cn )

College of Biological Sciences, China Agricultural University

\section{Article}

Keywords: sphingolipids, LCB1

Posted Date: December 29th, 2020

DOI: https://doi.org/10.21203/rs.3.rs-125529/v1 
License: (c) (i) This work is licensed under a Creative Commons Attribution 4.0 International License. Read Full License 
Title: Phosphorylation of LCB1 subunit of serine palmitoyltransferase stimulates its activity and modulates sphingolipid biosynthesis in Arabidopsis

Running title: Phosphorylation of LCB1 subunit activates serine palmitoyltransferase

Authors and affiliations: Yuan $\mathrm{Li}^{1, \dagger}$, Hanwei $\mathrm{Cao}^{1, \dagger}$, Tingting Dong ${ }^{1}$, Xiaoke Wang ${ }^{2}$, Liang $\mathrm{Ma}^{1}, \mathrm{Kun} \mathrm{Li}^{3}$, Huiqiang $\mathrm{Lou}^{2}$, Chunpeng Song ${ }^{3}$, and Dongtao $\operatorname{Ren}^{1 *}$

1 State Key Laboratory of Plant Physiology and Biochemistry, College of Biological Sciences, China Agricultural University, Beijing 100193, China

2 State Key Laboratory of Agro-Biotechnology and Beijing Advanced Innovation Center for Food Nutrition and Human Health, College of Biological Sciences, China Agricultural University, Beijing 100193, China

3 Collaborative Innovation Center of Crop Stress Biology, Henan Province. Institute of Plant Stress Biology, School of Life Science, Henan University, Kaifeng 475001, China

${ }^{\dagger}$ The authors contributed equally to this work

* Corresponding author: Dongtao Ren. E-mail address: ren@,cau.edu.cn 


\section{SUMMARY}

Sphingolipids are the structural elements for membrane lipid bilayers and the signal molecules for many cellular processes. Serine palmitoyltransferase (SPT) is the first committed and rate-limiting enzyme in the de novo sphingolipids biosynthetic pathway. The core SPT was previously suggested as a heterodimer consisting of LCB1 and LCB2 subunits. The SPT activity was shown to be inhibited by orosomucoid proteins (ORMs) and stimulated by small subunits of SPT (ssSPT). However, whether LCB1 is modified and how the modification regulates SPT activity have been unclear. Here, we show that activation of MPK3 and MPK6 by upstream MKK9 and Flg22 (a pathogen-associated molecular pattern) treatment increases SPT activity and induces the accumulation of sphingosine long-chain base (LCB) t18:0 in Arabidopsis thaliana; the activated MPK3 and MPK6 phosphorylate AtLCB1. Phosphorylation of AtLCB1 strengthens its binding with AtLCB2b, promotes its binding with ssSPTs, and stimulates the higher-orderoligomer and active SPT complexes formation. Our findings suggest a novel regulatory mechanism of SPT activity. 


\section{Introduction}

Sphingolipids, a class of natural lipids composed of a sphingoid base backbone (sphingosine), are ubiquitous in all eukaryotes and a few species of prokaryotes. Sphingosine N-acylated with a fatty acid (FA) forms ceramide (Cer). Through the addition of a variety of charged, neutral, glycosylated, or phosphorylated moieties, ceramides further form hundreds of complex sphingolipid species. Sphingolipids typically represent about 10 to $20 \%$ of cellular lipids and serve either as structural components of membranes modulating the physical properties of lipid bilayers and the activity of transmembrane proteins ${ }^{1,2}$ or as the signal molecules regulating various physiological processes and stress responses ${ }^{3-8}$.

The main aspects of sphingolipids biosynthesis and metabolism in eukaryotes are largely conserved, however, these compounds also show considerable diversity, as suggested by the finding that many sphingolipid structural modifications exist only in plants ${ }^{8,9}$. Most of the current knowledge about sphingolipids derives from research in animals and yeast. Apart from their structural roles, functions of sphingolipids in signaling plant (mostly in Arabidopsis thaliana based on mutant and chemical treatment analysis) development and stress responses have recently been reported, but the mechanisms are far from clear. Analysis of sphingosine kinase (SPHK) mutant $(s p h k)$ plants and of the effects of treatment with sphingosine-1-phosphate (the product of SPHK) indicate that sphingolipids are involved in abscisic acid-dependent regulation of stomatal closure ${ }^{10}$. The cell death phenotypes of ceramide kinase mutant (acd5) plants ${ }^{11}$ and ceramide synthase ( $\left.\mathrm{LOH} 2\right)$-overexpressing plants ${ }^{12}$, the early senescence phenotype of ORM knockdown mutants ${ }^{13}$, and cell division and growth phenotypes of LOH1- and $L O H 3$-overexpressing plants ${ }^{12}$ and of $L C B 2$ s knockdown plants indicate that sphingolipids mediate the regulation of cell growth and cell death processes. Defects of pollen formation in $l c b 2 a / l c b 2 b$ and ssspta mutants ${ }^{14}$, embryo development in $l c b 1$ mutants ${ }^{15}$, and reproductive growth transition in sbh1/sbh2 mutants ${ }^{16}$ reveal that sphingolipids are required for reproductive processes. Finally, the reduced sensitivity of $l c b k 2$ mutants to cold stress ${ }^{17}$, the enhanced tolerance of $A t c l b$ mutants to salt and drought ${ }^{18}$, and the identification of GIPC sphingolipids as NLP toxin receptors 
${ }^{19}$ suggest that sphingolipids also have roles in signaling responses to abiotic and biotic stresses.

The pathway of sphingolipids de novo synthesis starts with the condensation of serine and palmitoyl CoA into 3-ketodihydrosphingosine, which is catalyzed by a serine palmitoyltransferase (SPT). All known eukaryotic SPT core enzymes are suggested as endoplasmic reticulum (ER)-membrane-associated heterodimers composed of LCB1 and LCB2 subunits ${ }^{15,20}$. Loss of SPT activity is lethal ${ }^{15,21,22}$. SPT is believed to be the key point of sphingolipid biosynthesis regulation. Orosomucoid proteins (ORMs) and small subunits of SPTs (such as ssSPTs in animals and plants, and Tsc3p in yeast) are the currently known major regulators of SPT activity. By interacting with LCB1 and LCB2, ssSPTs or Tsc3p stimulate ${ }^{23-25}$ and ORMs inhibit SPT activity ${ }^{26-28}$. In yeast, phosphorylation of ORMs by the Ypk1 protein kinase interferes with their interaction to reverse the inhibition ${ }^{29}$; however, the phosphorylation domain does not exist in the ORMs of animals and plants ${ }^{30}$. The recent finding that Arabidopsis ORMs interact with SSSPT to inhibit SPT activity suggests another way that ORM could regulate SPT activity ${ }^{13}$. Considering the importance of sphingolipids and the irreplaceable role of SPT in sphingolipids biosynthesis, it seems plausible that the mechanisms regulating SPT activity may not be as simple as the ORM and ssSPT regulation. Indeed, phosphorylation of LCB2 by an unknown kinase in HeLa and HEK293 cells ${ }^{31,32}$ and phosphorylation of LCB1 by BCR-ABL kinase in chronic myeloid leukemia cells each inhibit SPT activity ${ }^{33}$. This implies that phosphorylation of LCB1 and LCB2 may also be a way to regulate SPT activity. However, the universality of LCB1 and LCB2 phosphorylation, the kinase(s) responsible for the phosphorylation, and the effects of their phosphorylation on SPT activity regulation are unknown.

Mitogen-activated protein kinase (MAPK) cascades, highly conserved signaling modules in eukaryotes, are composed of MAPKKK, MAPKK, and MAPK (known as MKKK, MKK, and MPK, respectively, in plants according to systemic nomenclature) ${ }^{34}$. After receiving signals from receptors or sensors, MAPK cascades are activated through sequential phosphorylation of the three kinases. The activated MAPKs, in turn, phosphorylate their targets to mediate the regulation of cellular processes. MAPK 
cascades have been shown to play important roles in signaling plant growth, development, and stress responses. Arabidopsis MPK3 and MPK6 are the highly studied plant MAPKs to date. Upon activation by different upstream MKKs, MPK3 and MPK6 perform distinct functions. For example, MKK2-MPK4/MPK6 regulates cold and salt stress responses ${ }^{35}$; MKK3-MPK6 mediates jasmonic acid signaling ${ }^{36}$; MKK4and/or MKK5-MPK3/MPK6 regulates reactive oxygen species signaling ${ }^{37,38}$, cold, abscisic acid, and defense responses ${ }^{39-42}$, and stomata and ovule development ${ }^{43,44}$; MKK7-MPK6 regulates polar auxin transport ${ }^{45}$; MKK4-, MKK5-, and/or MKK9MPK3/MPK6 regulate ethylene and camalexin biosynthesis 46,47, phosphate homeostasis, and ethylene signaling ${ }^{48,49}$; and MKK10-MPK6 regulates the red light response ${ }^{50}$. Two recent studies showing that exogenous LCB treatment activates MPK6 imply that the MAPK cascade is involved in sphingolipids signaling transduction ${ }^{17,51}$; however, whether and how a particular MAPK cascade regulates sphingolipids biosynthesis are not known.

Here, we show that after activation by upstream MKK9 and Flg22 (one of the microbeassociated molecular patterns) treatment, MPK3 and MPK6 phosphorylate AtLCB1; AtLCB1 phosphorylation then leads to the formation of high-oligomer and highactivity SPT and subsequently to increased-free LCB t18:0 accumulation. Our results suggest that phosphorylation of AtLCB1 is a previously unknown mechanism of SPT activity regulation in sphingolipids biosynthesis.

\section{Results}

Activation of MKK9-MPK3/MPK6 induces sphingolipids biosynthesis in transgenic plants.

In this study, we initially analyzed the profiles of methanol-extracted metabolites from the inducible promoter controlled $M K K 9^{D D}$ (a kinase-active form of MKK9) transgenic seedlings before (-DEX) or after (+DEX) the transgene induction. Surprisingly, except for camalexin that we have reported previously ${ }^{47,52}$, a second strongly induced compound was found in the extract from $M K K 9^{D D}$ seedlings after MKK9 ${ }^{\mathrm{DD}}$ induction (+DEX); this compound is further identified as 4-hydroxysphinganine (LCB t18:0), a 
major species of the long-chain bases that is the basal backbone of sphingolipids in plants (Figure 1a). This finding leads a speculation that MKK9 may regulate sphingolipids homeostasis.

We then conducted a sphingolipidomic analysis by extraction with an established sphingolipids extraction procedure and detection with an Exion UPLC system coupled to a triple quodrupole/ion trap mass spectrometer (QTRAP 6500 Plus, Sciex) (Figure 1b). The results showed that all four major classes of sphingolipids (ceramides, hydroxyceramides, GlcCers, and GIPCs) along with free LCB(P)s (LCBs and phosphorylated LCBs) were identified in $M K K 9^{D D}$ seedlings either before or after MKK $^{\mathrm{DD}}$ induction. Comparison showed that total amounts of free $\operatorname{LCB}(\mathrm{P}) \mathrm{s}$ and ceramides were significantly increased in $M K K 9^{D D}$ seedlings after $\mathrm{MKK} 9^{\mathrm{DD}}$ induction (+DEX), of about 5-fold and 1.8-fold of that in seedlings before MKK9 ${ }^{\mathrm{DD}}$ induction (DEX), respectively, while total amounts of hydroxyceramides, GIPCs, and glucosylceramides in $M K K 9^{D D}$ seedlings before (-DEX) or after (+DEX) MKK9 $9^{\mathrm{DD}}$ induction did not show significantly difference. Further analysis of individual species in all classes of sphingolipids revealed that some free $\mathrm{LCB}(\mathrm{P}) \mathrm{s}(\mathrm{t} 18: 0-\mathrm{P}, \mathrm{t} 18: 1$, and t18:0) and most of the t18:0-containing ceramides were strongly increased, while some t18:1-containing glucosylceramides (h22:0, h24:1, h26:1, and h26:0) were slightly decreased in $M K K 9^{D D}$ seedlings after MKK9 ${ }^{\mathrm{DD}}$ induction (+DEX). Notably, the amounts of free LCB t18:0 and t18:0/c16:0 ceramide in $M K K 9^{D D}$ seedlings after MKK $9^{\mathrm{DD}}$ induction (+DEX) were 9.4-fold and 11.6-fold higher than that in $M K K 9^{D D}$ seedlings before MKK9 $9^{\mathrm{DD}}$ induction (-DEX), respectively. The over-accumulation of free LCB t18:0 and t18:0-containing ceramides after MKK9 ${ }^{\mathrm{DD}}$ induction suggests that the activation of MKK9 activates sphingolipids biosynthetic pathway. Since, LCBs contents are generally used to reflect the amount of sphingolipids ${ }^{15}$, to simplify further description, LCBs in seedlings were measured using HPLC as described by Chen et al (Figure S1) ${ }^{16}$, and only the contents of free LCB t18:0 were presented in our later contexts.

Two independent homozygous transgenic lines for $M K K 9^{D D}$ were further analyzed, and two $M K K 9^{K R}$ (a kinase-inactive form of MKK9) transgenic lines (lines 178 and B106) 
and one empty vector transgenic line (as Vector) were used as controls. The results showed that $M K K 9^{D D}$ seedlings (lines 129 and B63) produced much higher levels of free LCB t18:0 than $M K K 9^{K R}$ and Vector seedlings, which produced comparable and relatively low levels of free LCB t18:0; the levels of free LCB t18:0 in $M K K 9^{D D}$ seedlings were 12-fold higher for line 129 and 17-fold higher for line B63 than Vector seedlings (Figure 1c). Kinase activity assay showed the strong activation of MPK3 and MPK6 in $M K K 9^{D D}$ transgenic lines (Figure S2a). The requirement of MPK3 and MPK6 activities for the induction of free LCB t18:0 was further analyzed using $M K K 9^{D D}$, $M K K 9^{D D} / m p k 3$, and $M K K 9^{D D} / m p k 6$ seedlings. MKK9 ${ }^{\mathrm{DD}}$ induction in all three genotypes could induce an elevation of free LCB t18:0, however, $M K K 9^{D D} / m p k 3$ and $M K K 9^{D D} / m p k 6$ seedlings produced nearly 70\% lower levels of free LCB t18:0 compared with $M K K 9^{D D}$ seedlings at $4 \mathrm{~h}$ after $M K K 9^{D D}$ induction (Figure 1d). Immunoblotting and kinase activity assays revealed the complete loss of MPK3 activity in $M K K 9^{D D} / m p k 3$ seedlings and MPK6 activity in $M K K 9^{D D} / m p k 6$ seedlings (Figure $\mathrm{S} 2 \mathrm{~b})$. These results suggest that activation of MKK9-MPK3/MPK6 in seedlings activates sphingolipids biosynthesis.

\section{Flg22 induces LCB t18:0 elevation through activating MKK9-MPK3/MPK6.}

MPK3 and MPK6 are known previously as two stresses-activated MAPKs in Arabidopsis $35,36,41,46,49,50,53,54$. To explore whether and which stresses could activate the two kinases and subsequently activate sphingolipids pathway, LCB t18:0 contents and kinase activities in Arabidopsis seedlings under a variety of stimuli were analyzed. The results showed that compared with the level in untreated seedlings (Control), Flg22 and $\mathrm{H}_{2} \mathrm{O}_{2}$ treatments significantly stimulated the increases of free LCB t18:0, of about $360 \%$ and $140 \%$, respectively, but $\mathrm{NaCl}$ and cold $\left(4^{\circ} \mathrm{C}\right)$ treatments did not alter the free $\mathrm{LCB}$ t18:0 contents (Figure 2a). Kinase activity assays showed the strong activation of 46and 43-kD kinases by Flg22 treatment and of 46-kD kinase by $\mathrm{H}_{2} \mathrm{O}_{2}$ treatment, but only weak activation of the $46-\mathrm{kD}$ kinase and moderate activation of a $40-\mathrm{kD}$ kinase by $\mathrm{NaCl}$ and cold treatments (Figure 2b). Analyses of kinase activities and LCB t18:0 levels in seedlings of $m p k 3$ and $m p k 6$, the knockout mutants of MPK3 and MPK6, showed that 
although Flg22 treatment still induced the increasing of free LCB t18:0 contents in $m p k 3$ and mpk6 seedlings, however, the degree of induction in mpk3 and mpk6 seedlings were significantly suppressed: $m p k 3$ and $m p k 6$ seedlings treated with Flg22 produced $30 \%$ and $45 \%$ less free LCB t18:0, respectively, than Col-0 seedlings (Figure 2c). The concurrent disappearance of 43-kD kinase activity in $m p k 3$ seedlings and 46$\mathrm{kD}$ kinase activity in $m p k 6$ seedlings treated with Flg22 confirmed that the 43- and 46kD kinases were MPK3 and MPK6, respectively (Figure 2d). Since MKK9 was showed to induce the increasing of LCB t18:0 in transgenic seedlings by activating MPK3 and MPK6, a T-DNA insertion MKK9-null mutant line ( $m k k 9)$ was also analyzed. The results revealed that the level of free LCB t18:0 elevation induced by Flg22 was significantly suppressed in $m k k 9$ seedlings, so that the mutant seedlings produced about 30\% less free LCB t18:0 than Col-0 seedlings (Figure 2e). The kinase activities of MPK3 and MPK6 induced by Flg22 were significantly compromised in $m k k 9$ seedlings compared with Col-0 seedlings (Figure 2f). These results suggest that Flg22 treatment induces the elevation of free LCB t18:0 through activating MKK9-MPK3/MPK6 cascade.

\section{MKK9-MPK3/MPK6 induces LCB t18:0 via activating its de novo synthesis.}

Sphingolipids catabolism and LCB de novo synthesis are the major pathways influencing the cellular free LCB pool. To assess which of these pathways is predominantly required for the MAPK cascade-induced free LCB t18:0, we compared the free LCB t18:0 levels produced in $M K K 9^{D D}$ and $M K K 9^{D D} / l c b 2 a / l c b 2 b i$ seedlings. The $l c b 2 a / l c b 2 b i$ line is a previously used double mutant, that AtLCB2a is knocked out $(l c b 2 a)$ by T-DNA insertion and $A t L C B 2 b$ is knocked down $(l c b 2 b i)$ by an inducible small interfering RNA (siRNA) expression (induced by methoxyfenozide [Meth]), and the de novo biosynthesis of LCBs in this mutant is significantly reduced after Meth application $^{14}$. After MKK9 ${ }^{\mathrm{DD}}$ induction (+ DEX), MKK9DD/lcb2a/lcb2bi seedlings produced $62 \%$ less of free LCB t18:0 than $M K K 9^{D D}$ seedlings in the absence of Meth (+ DEX, - Meth), and 90\% less of free LCB t18:0 than $M K K 9^{D D}$ seedlings in the presence of Meth (+DEX, +Meth) (Figure 3). This near abolishment of the MAPK- 
cascade-induced free LCB t18:0 elevation in $M K K 9^{D D} / l c b 2 a / l c b 2 b i$ seedlings suggests that the elevated free LCB t18:0 is generated through the LCB de novo biosynthetic pathway.

\section{MKK9-MPK3/MPK6 interacts and phosphorylates AtLCB1.}

To explore the mechanism of MAPK-cascade-induced LCB t18:0 biosynthesis, we first used quantitative PCR to detect the transcription of the genes encoding the enzymes (including SPT, KSR, and SBH ) and their regulators (ORMs and ssSPT) controlling the conversion of serine and palmitoyl CoA to LCB t18:0 ${ }^{9}$. We adapted a change of more than 2-fold as indicating significant induction or reduction. The results revealed that $A t L C B 1$ was significantly induced in Col-0, $m p k 3, m p k 6$, and $m k k 9$ seedlings after Flg22 treatment, although its induction in $m p k 3, m p k 6$, and $m k k 9$ seedlings was a little suppressed compared with that in Col-0; $A t L C B 2 b$ was also significantly induced after Flg22 treatment, to a comparable level in all four genotypes; $s S S P T a$ and $s S S P T b$ were unaltered in Col-0 and mpk6 seedlings but significantly reduced in $m p k 3$ and $m k k 9$ seedlings after Flg22 treatment; the transcription of ORM1, ORM2, TSC10A, TSC1OB, $S B H 1$, and $S B H 2$ did not show significant change among the four genotypes (Figure S3a). Analysis of the transcription of these genes in the MKK9 transgenic variants $M K K 9^{D D}$ and $M K K 9^{K R}$, and crosses of $M K K 9^{D D}$ with $m p k 3$ and $m p k 6$, showed that of $A t L C B 1$ and $A t L C B 2 b$ but suppressed that of TSC1OB and SSSPTa, while MKK ${ }^{\mathrm{KR}}$ induction (+DEX) in $M K K 9^{K R}$ seedlings did not alter the transcription of any of these genes. The induction of $A t L C B 1$ and reduction of $T S C 10 B$ were partially suppressed in $M K K 9^{D D} / m p k 3$ and $M K K 9^{D D} / m p k 6$ seedlings, and the induction of $A t L C B 2 b$ was unaffected in $M K K 9^{D D} / m p k 3$ seedlings and suppressed in $M K K 9^{D D} / m p k 6$ seedlings (Figure S3b). The results suggest that transcription of the various genes in the LCB t18:0 biosynthetic pathway is differentially regulated by MKK9-MPK3/MPK6.

Next, we analyzed the contribution of MKK9-MPK3/MPK6-regulated genes transcription to the elevation of free LCB t18:0 level by comparing free LCB t18:0 contents in $M K K 9^{D D}$ seedlings treated with actinomycin D (ActD), an inhibitor of gene 
transcription, to those in untreated controls. MPK3 and MPK6 were effectively activated in $M K K 9^{D D}$ seedlings by $1 \mathrm{~h}$ after MKK $9^{\mathrm{DD}}$ induction (Figure S2b), whereas $A t L C B 1$ and $A t L C B 2 b$ transcription and free LCB t18:0 accumulation were not yet induced at that time point (Figure S4a and Figure 1d), we therefore started the ActD treatment $1 \mathrm{~h}$ after DEX addition. The results showed that MKK9-MPK3/MPK6induced transcription of $A t L C B 1$ and $A t L C B 2 b$ was effectively blocked (Figure S4b), and MPK3 and MPK6 were activated substantially in $M K K 9^{D D}$ seedlings in the presence of ActD plus DEX (Figure S4c). However, $M K K 9^{D D}$ seedlings treated with ActD plus DEX accumulated similar levels of free LCB t18:0 compared to the seedlings treated with DEX alone (Figure S4d). These results suggest that MKK9-MPK3/MPK6induced free LCB t18:0 accumulation does not rely on the transcriptional regulation of LCB biosynthetic genes.

We further analyzed the possibility that MKK9-MPK3/MPK6 phosphorylates free LCB t18:0 de novo biosynthetic enzymes or an assortment of their regulators. We used a yeast two-hybrid ( $\mathrm{Y} 2 \mathrm{H})$ assay to initially screen the candidate of MPK3 or MPK6 targets in these proteins. Among the proteins tested, AtLCB1 and the two ssSPTs (ssSPTa and ssSPTb) interacted with MPK3 and MPK6, as indicated by a 3-AT containing synthetic drop-out (SD) medium growth assay with $\beta$-galactosidase visualization (Figure 4a). Since ssSPTs did not contain a putative MAPK phosphorylation site, we therefore selected AtLCB1 for further study. Then, we tested the interaction between MPK3/MPK6 and AtLCB1 using a split bimolecular complementation (split-BiFC) assay. We detected YFP fluorescence signals when AtLCB1-YFP NE was co-expressed with either MPK3-YFP CE or MPK6-YFP CE, whereas expression of AtLCB1-YFP NE or MPK3-YFP CE or MPK6-YFP CE alone did not yield detectable YFP fluorescence (Figure 4b). These results suggest that AtLCB1 may be a target of MPK3 and MPK6.

We further investigated the in vitro phosphorylation of AtLCB1 by activated MPK3 and MPK6. In-solution kinase assays showed that MKK9-activated MPK6 strongly and MPK3 very weakly phosphorylated $\triangle \mathrm{AtLCB} 1^{\mathrm{WT}}$, a truncated AtLCB1 protein with its putative transmembrane region (1-60 amino acid residues) deleted. 
Similar assays of mutants with Thr169 and/or Ser398, the putative MAPK phosphorylation sites in $\triangle \mathrm{AtLCB} 1^{\mathrm{WT}}$ protein, replaced by Ala revealed a strong reduction in the phosphorylation of $\triangle \mathrm{AtLCB} 1^{\mathrm{T} 169 \mathrm{~A}}$ and $\triangle \mathrm{AtLCB} 1^{\mathrm{T} 169 \mathrm{~A} / \mathrm{S} 398 \mathrm{~A}}$ and moderately reduction in that of $\triangle \mathrm{AtLCB} 1^{\mathrm{S} 398 \mathrm{~A}}$ phosphorylation (Figure $4 \mathrm{c}$ ). These results suggest that MKK9-MPK3/MPK6 phosphorylates AtLCB1 protein in vitro, and that Thr169 and Ser398 are the potential phosphorylation sites.

For in vivo AtLCB1 phosphorylation assays, protein samples from seedlings were separated by SDS-PAGE or Phos-tag gel, and AtLCB1 was detected by immunoblotting using an AtLCB1-specific antibody raised in this study (Figure S5). The antibody detected a single AtLCB1 band in each sample from Col-0, mpk3, and mpk6 seedlings with or without Flg22 treatment, and from $M K K 9^{D D}$ transgenic and crossed seedlings with or without MKK $9^{\mathrm{DD}}$ induction, after separation by SDS-PAGE (Figure 4d). However, three AtLCB1 bands were detected in each sample from seedlings of all genotypes after separation on a Phos-tag gel. When the samples from Flg22-treated Col-0 seedlings and $\mathrm{MKK} 9^{\mathrm{DD}}$-induced $M K K 9^{D D}$ seedlings were incubated with alkaline phosphatase (+ CIAP), the single AtLCB1 band separated by SDS-PAGE showed a slight downshift; furthermore, the two upper AtLCB1 bands separated by the Phos-tag gel were missing and the bottom AtLCB1 band showed increased intensity. These results suggest that two upper bands are the phosphorylated forms of AtLCB1 and that both phosphorylated (the two upper bands) and unphosphorylated (the bottom band) AtLCB1 exist in seedlings. Analysis of the ratio of phosphorylated AtLCB1 to total AtLCB1 revealed that Flg22 treatment and MKK $9^{\mathrm{DD}}$ induction each increased the levels of phosphorylated AtLCB1 and that loss of either MPK3 or MPK6 function lessened this increase. The results suggest that MPK3 and MPK6 activated by either Flg22 or MKK9 phosphorylates AtLCB1 in vivo.

\section{Phosphorylation of AtLCB1 enhances SPT activity.}

Since, AtLCB1, one of the core subunits of SPT, is phosphorylated by MKK9MPK3/MPK6, we further explored the effects of AtLCB1 phosphorylation on SPT activity. The results showed that SPT activities in Col-0, mkk9, mpk3, and mpk6 
seedlings in the absence of Flg22 were all comparable and that they all increased upon the Flg22 treatment, but to different degrees: the levels in $m k k 9, m p k 3$, and $m p k 6$ seedlings were $17 \%, 10.5 \%$, and $20 \%$ lower, respectively, than that in Col-0 (Figure 5a). The SPT activities in $M K K 9^{D D}, M K K 9^{D D} / \mathrm{mpk3}$, and $M K K 9^{D D} / m p k 6$ seedlings were all significantly increased upon MKK9 ${ }^{\mathrm{DD}}$ induction (+DEX) compared with those seen in the absence of MKK9 $9^{\mathrm{DD}}$ induction (-DEX), but the +DEX levels in $M K K 9^{D D} / m p k 3$ and $M K K 9^{D D} / m p k 6$ seedlings were $18 \%$ and $28 \%$ lower than that in $M K K 9^{D D}$ seedlings, respectively (Figure 5b). The correlation between SPT activity and AtLCB1 phosphorylation (Figure $4 \mathrm{c}$ and $4 \mathrm{~d}$ ) suggests that phosphorylation of AtLCB1 may activate SPT activity.

We initially analyzed the effect of AtLCB1 phosphorylation on SPT activity using a yeast $l c b 1$ mutant growth rescue assay by expressing AtLCB1 variants in $l c b 1$ mutant. Since a previous report had shown that restoring SPT activity in the yeast $l c b 1$ mutant required the co-expression of AtLCB1 and AtLCB2 ${ }^{15}$, we also adopted this strategy in our experiments. Co-expression of AtLCB1 ${ }^{\mathrm{WT}}$ or AtLCB1 ${ }^{\mathrm{DD}}$ (in which both Thr169 and Ser398 are mutated to Asp to mimic phosphorylated AtLCB1) with AtLCB2b in the yeast $l c b 1$ mutant rescued the mutant's growth-deficient phenotype, whereas coexpression of the nonphosphorylatable variant AtLCB1 ${ }^{\mathrm{AA}}$ (in which both Thr169 and Ser398 are mutated to Ala) with AtLCB2b did not (Figure 5c). Moreover, when we added PD98059, an inhibitor of MAPKK activity, to the growth media to block MAPKs activation, the ability of AtLCB $1^{\mathrm{WT}}$ and AtLCB2b to rescue the yeast $l c b 1$ mutant growth phenotype was clearly lost. Immunoblotting results confirmed the successful expression of AtLCB1 variants and AtLCB2b in the transformed $l c b 1$ cells (Figure S6). The results suggest that phosphorylation of AtLCB1 is required for the SPT activity in yeast cells.

Transgenic Arabidopsis plants carrying MYC-tagged AtLCB1 variants encoding genes under the control of the $35 \mathrm{~S}$ promoter or the steroid-inducible promoter were tried to generate to investigate the functions of these variants in plants ${ }^{55}$; however, no $A t L C B 1^{D D}$ transgenic plants obtained expressed detectable MYC-AtLCB1 ${ }^{\mathrm{DD}}$ proteins. To fill this gap, we set out to generate transgenic plants with MYC-AtLCB1 variant 
encoding genes by the native $A t L C B 1$ promoter, and we selected the two homozygous lines for each genotype with the highest MYC-AtLCB1 protein levels for further analysis. In the absence of Flg22 treatment, very low and comparable amounts of free LCB t18:0 accumulated in Col-0 and AtLCB1 variants transgenic seedlings; upon Flg22 treatment, accumulation of free LCB t18:0 in all genotype seedlings significantly increased, but the amounts of free LCB t18:0 in the Flg22-treated MYC-AtLCB1 ${ }^{A A}$ seedlings was only half of that in Col-0 wild-type, or MYC-AtLCB1 ${ }^{W T}$, or $M Y C$ $A t L C B 1^{D D}$ seedlings (Figure 5d). In addition, SPT activities in Col-0 and AtLCB1 variants transgenic seedlings treated with Flg22 were significantly induced; however, SPT activities in both of the untreated and Flg22-treated $M Y C$-AtLCB1 ${ }^{A A}$ seedlings were only half of those in Col-0, or $M Y C-A t L C B 1^{W T}$, or $M Y C-A t L C B 1^{D D}$ seedlings. When we crossed $M Y C-A t L C B 1^{A A}$ and $M Y C-A t L C B 1^{W T}$ plants into the $M K K 9^{D D}$ background, we found that without MKK $9^{\mathrm{DD}}$ induction (-DEX), similar lower levels of free LCB t18:0 accumulated in $M K K 9^{D D}$ and crossed seedlings, and free LCB t18:0 was significantly increased upon $\mathrm{MKK} 9^{\mathrm{DD}}$ induction (+DEX) in both groups of seedlings; however, $M Y C$-AtLCB1 $1^{A A} / M K K 9^{D D}$ seedlings accumulated much less free LCB t18:0 than $M K K 9^{D D}$ or $M Y C-A t L C B 1^{W T} / M K K 9^{D D}$ seedlings with MKK9 $9^{\mathrm{DD}}$ induction (+DEX). SPT activities in $M Y C-A t L C B 1^{A A} / M K K 9^{D D}$ seedlings with or without MKK9 $9^{\mathrm{DD}}$ induction (-DEX or +DEX) were significantly impaired compared with those in $M K K 9^{D D}$ or $M Y C-A t L C B 1^{W T} / M K K 9^{D D}$ seedlings (Figure 5e). The dominant-negative role of MYC-AtLCB1 ${ }^{\mathrm{AA}}$ in regulating SPT activity in vivo further confirmed the importance of phosphorylated AtLCB1 for SPT activity.

\section{Phosphorylation of AtLCB1 stimulates higher-order and active SPT complex} formation.

To explore why the expression of nonphosphorylatable MYC-AtLCB1 ${ }^{\mathrm{AA}}$ in planta regulates SPT activity in a dominant-negative fashion, we decided to test interactions between AtLCB1 variants or the variants with other subunits in SPT complex using $\mathrm{Y} 2 \mathrm{H}$ assay in the presence or absence of PD98059. We found that AtLCB1 variants interacted with each other in either the presence or the absence of PD98059 (Figure 6a); 
in the absence of PD98059, AtLCB $1^{\mathrm{WT}}$ and AtLCB1 ${ }^{\mathrm{DD}}$ interacted normally with AtLCB2b and ssSPTs, while AtLCB1 ${ }^{\mathrm{AA}}$ interacted very weakly with AtLCB2b but not at all with ssSPTs; however, in the presence of PD98059, the interaction between AtLCB $1^{\text {WT }}$ and AtLCB2b or ssSPTs was almost abolished, while the interactions between AtLCB1 ${ }^{\mathrm{DD}}$ and AtLCB2b or ssSPTs were not affected. These results imply that the SPT complex may contain multiple phosphorylated and/or unphosphorylated

AtLCB1 subunits, but because unphosphorylated AtLCB1 lacks the ability to bind AtLCB2 and ssSPTs, its presence in the SPT complex reduces SPT enzyme activity. To further explore the effects of AtLCB1 phosphorylation on SPT activity, we separated protein samples extracted from $M K K 9^{D D}$ seedlings with (+DEX) or without (-DEX) MKK9 ${ }^{\text {DD }}$ induction by Superdex-200 10/300 GL gel-filtration chromatography and analyzed the resulting fractions. AtLCB1 proteins were found in fractions 5-12 (corresponding to a protein complex size of $600 \mathrm{kD}-150 \mathrm{kD}$ ) in samples without MKK $^{\text {DD }}$ induction and in fractions 5 to 10 (corresponding to a protein complex size of $600 \mathrm{kD}-200 \mathrm{kD}$ ) in samples with MKK9 ${ }^{\mathrm{DD}}$ induction by immunoblotting detection after separation on SDS-PAGE gels (Figure 6b). When we repeated the process but with separation on Phos-tag gels, we found that the proportions of phosphorylated AtLCB1 reduced with the molecular sizes of the SPT complexes decreased. At the extremes, the $600-\mathrm{kD}$ SPT complex (in fraction 5) contained only the phosphorylated AtLCB1, and complexes smaller than 200-kD (in fractions 10-12) contained only the nonphosphorylated AtLCB1. The proportion of higher-molecular-size SPT was higher in seedlings with $\mathrm{MKK} 9^{\mathrm{DD}}$ induction than in seedlings without MKK9 ${ }^{\mathrm{DD}}$ induction. Next, we compared the SPT activities in fraction 5 (with the higher proportion of phosphorylated AtLCB1), fraction 7 (with a moderate ratio), and fraction 10 (with a very low ratio) from sample of $M K K 9^{D D}$ seedlings with or without MKK9 ${ }^{\mathrm{DD}}$ induction, using the same levels of total AtLCB1 protein. SPT activities correlated well with the ratio of AtLCB1 phosphorylation in all tested fractions: SPT activities for fraction 10 from both samples were comparably lower, about $0.055 \mathrm{pmol} / \mathrm{min}$; SPT activities for fraction 7 from samples with or without the MKK9 $9^{\mathrm{DD}}$ induction were 0.125 and 0.097 $\mathrm{pmol} / \mathrm{min}$, respectively; and SPT activities for fraction 5 from samples with or without 
the MKK $9^{\mathrm{DD}}$ induction were 0.227 and $0.173 \mathrm{pmol} / \mathrm{min}$, respectively (Figure $6 \mathrm{c}$ ). These results suggest that phosphorylation of AtLCB1 is required for the formation of higherorder oligomers and active SPT complexes.

We also analyzed proteins from $M Y C-A t L C B 1^{D D}$ (line 11) and $M Y C-A t L C B 1^{A A}$ (line 1) seedlings treated with Flg22 using the same electrophoresis and immunoblotting procedures as aforementioned. Notably, MYC-AtLCB1 ${ }^{\mathrm{DD}}$ proteins were preferentially present, compared to MYC-AtLCB1 ${ }^{\mathrm{AA}}$ proteins, in highermolecular-size SPT complexes (Figure 6d). We then compared the SPT activities in fraction 7 with similar ratios of phosphorylated and unphosphorylated AtLCB1 native proteins, as well as very lower levels of MYC-AtLCB1 ${ }^{\mathrm{AA}}$ or MYC-AtLCB1 ${ }^{\mathrm{DD}}$ proteins from $M Y C-A t L C B 1^{A A}$ and $M Y C-A t L C B 1^{D D}$ seedlings, using the same levels of total AtLCB1 protein. The SPT activity of fraction 7 from $M Y C-A t L C B 1^{D D}$ seedlings was $169 \%$ that of the same fraction from $M Y C-A t L C B 1^{A A}$ seedlings (Figure 6e). This further confirmed that AtLCB1 phosphorylation is required for SPT activity.

\section{Discussion}

As the enzyme that catalyzes the first committed and rate-limiting step in the sphingolipids biosynthetic pathway, SPT plays crucial roles in regulating sphingolipids homeostasis. ORM inhibition ${ }^{26,27,56,57}$ and ssSPT activation ${ }^{23-25}$ comprise the currently known mechanism for SPT activity regulation. Inhibition of SPT activity by ORM in yeast is reported to be accompanied by the increased of SPT dimerization or oligomerization, while the relief of ORM inhibition through its phosphorylation triggered a shift of the SPT complex toward monomeric organization ${ }^{58}$. The mechanisms by which ORM inhibition and inhibition relief in animals and plants are still unknown. It has been suggested that ssSPTs or TSC3 stimulate SPT activity, possibly by facilitating LCB1 and LCB2 interaction or stabilizing SPT ${ }^{23-25}$. The LCB2 subunit contains the active site that binds the pyridoxal phosphate cofactor, while LCB1 lacks a catalytic residue; however, data from structural modeling and mutational studies indicated that the active site of the SPT core lies at the interface of the heterodimeric enzyme, and residues in both subunits could be involved in the SPT catalytic process 
(Gable et al., 2002). Lack of either LCB1 or LCB2 proteins destabilizes the other complex members within cells, suggesting that SPT may be regulated by protein stability ${ }^{24,59}$. Recently, Ser384 of LCB2s in HeLa and HEK293 were found to be phosphorylated, and a phosphomimic mutation was seen to decrease the SPT activity on the substrate L-serine ${ }^{31,32}$. Moreover, Tyr164 of LCB1 in chronic myeloid leukemia cells was reported to be phosphorylated by BCR-ABL, a chimeric tyrosine kinase encoded by a fusion gene that is newly created by the "Philadelphia translocation" during the development of chronic myeloid leukemia, to attenuate SPT activity ${ }^{33}$. Although the kinase responsible for LCB2s phosphorylation is currently unknown ${ }^{32}$, and BCR-ABL kinase is impossible to present in any other types of cells, these results imply that the phosphorylation of LCB1 and LCB2 may be involved in the regulation of SPT activity.

In this study, we initially demonstrated that activation of the MPK3/MPK6mediated MAPK cascade was required for PAMPs (e.g., Flg22)-elicited free LCB t18:0 accumulation. This conclusion is supported by the following two pieces of evidence: First, among all the stimuli used, Flg22 treatment most strongly activated MPK3/MPK6 and significantly induced free LCB t18:0 accumulation, and the elevation of the free LCB t18:0 abundance was compromised in the mpk3 and mpk6 mutants. Second, MKK $^{\mathrm{DD}}$ induction of MPK3 and MPK6 activation and free LCB t18:0 levels in $M K K 9^{D D}$ transgenic seedlings was significant, whereas in $M K K 9^{D D} / m p k 3$ and $M K K 9^{D D} / m p k 6$ seedlings was compromised. Our findings reveal interlinkage between MAPKs activation (e.g., well-documented MPK3 and MPK6 activation in Arabidopsis) 60,61 and pathogens-induced LCBs accumulation ${ }^{13,25,62-64}$.

LCB t18:0 is synthesized through three steps in the sphingolipids de novo synthesis pathway, catalyzed subsequently by SPT, KSR, and $\mathrm{SBH}^{7}$. MAPK-cascadesinduced LCB t18:0 accumulation could be controlled either by transcriptional regulation of the genes encoding these three enzymes and their regulators or by the phosphorylation-based regulation of the activity of one or more of proteins. Recent reports have shown that both up-regulation of SSSPT transcription ${ }^{25}$ and downregulation of $O R M$ transcription significantly increased SPT activity in Arabidopsis ${ }^{13,26}$; 
however, in our experiments, Flg22 treatment and MPK3/MPK6 activation by MKK9 did not reduce ORMs transcription or induce $s S S P T$ s transcription (Figure S3). Among the genes investigated, we observed up-regulation of transcription only for AtLCBIand $A t L C B 2 b$ genes after Flg22 treatment and MPK3/MPK6 activation by MKK9; however, treatment with the transcription inhibitor ActD compromised the increased transcription of the two genes but did not affect the elevation of free LCB t18:0 accumulation (Figure S4). This is not surprising because overexpression of LCB1 and LCB2, individually or in combination, has not previously been found to alter SPT activity with respect to the native expression ${ }^{25,65}$, even though the LCB1 and LCB2 are required for SPT activity in yeast, plant, and animal cells ${ }^{14,15,20}$. These results excluded the involvement of transcriptional regulation in controlling SPT activity and free LCB t18:0 accumulation.

Interestingly, we further discovered that AtLCB1 is a substrate of MPK3/MPK6 both in vitro and in vivo (Figure 4 and 5). In both of Flg22-treated and MKK9 ${ }^{\mathrm{DD}}$ induced transgenic seedlings, the AtLCB1 phosphorylation ratio, SPT activity, and free LCB t18:0 were found significantly elevated; SPTs are presented in various molecular sizes in seedlings in all genotypes, and as the size increased, the ratio of phosphorylated AtLCB1 and the SPT activity also increased (Figure 6). This led us to hypothesize that phosphorylation of AtLCB1 by the MKK9-MPK3/MPK6 cascade causes higher oligomerization of SPT that increases its activity. The results of expression of AtLCB1 variants in the yeast $l c b 1$ mutant and treatment with a MAPK kinase inhibitor showed that phosphorylation of AtLCB1 facilitates AtLCB2 and ssSPT binding. These data suggest that the phosphorylation of AtLCB1 serves as a novel mechanism of regulating SPT activity.

Based on results from this and previous studies, we propose a working model of SPT activity regulation (Figure 6f). When seedlings are grown under normal conditions, a lower proportion of phosphorylated AtLCB1 is present in SPT complexes, which thus exhibit basal SPT activity and maintain normal sphingolipids homestasis; upon stimulation by extracellular stimuli ( e.g., pathogens infection or other abiotic stresses) or intracellular signals (e.g., growth and development signals), MAPK cascades (e.g., 
MKKKx(?)-MKK9-MPK3/MPK6) are activated by an upstream receptor(s)/sensor(s); AtLCB1 is then phosphorylated by the active MPKs, and the phosphorylation of AtLCB1 strengthens the binding of AtLCB2 and ssSPT and enhances the formation of higher-order oligomers and higher-activity SPT complexes, which in turn increased de novo sphingolipids biosynthesis to meet the requirements. Future studies addressing how the phosphorylated AtLCB1, ssSPTs, and ORMs coordinately regulate SPT activity will help us to fully understand the mechanism of SPT activity regulation.

\section{Methods}

\section{Plant materials and growth conditions.}

Arabidopsis thaliana Col-0 wild-type, mutant, and transgenic seeds were surface sterilized. After treatment at $4{ }^{\circ} \mathrm{C}$ for 4 days, the seeds were germinated on $0.8 \%$ agar plates containing $0.5 \times$ Murashige and Skoog (MS), pH 5.7, and 1\% sucrose. Seedlings on plates were then transferred to soil and grown at $22^{\circ} \mathrm{C}$ in a growth room under a 16h-light $/ 8$-h-dark photoperiod at a photon flux density of $100 \mu \mathrm{mol} / \mathrm{m}^{2} / \mathrm{s}$. The seedlings grown in liquid culture medium were grown and treated as previously described ${ }^{47}$. For chemicals treatments, final concentrations of $0.2 \mu \mathrm{M}$ Flg22, $2 \mu \mathrm{M}$ Dexamethasone (DEX), $100 \mathrm{mM} \mathrm{NaCl}, 2 \mathrm{mM} \mathrm{H} \mathrm{O}_{2}, 100 \mu \mathrm{M}$ actinomycin D (ActD), and 1/10,000 diluted methoxyfenozide (Meth) were used, respectively. For cold treatment, seedlings were transferred to $4{ }^{\circ} \mathrm{C}$. Samples were collected, quick frozen in liquid nitrogen, and stored at $-80{ }^{\circ} \mathrm{C}$ until use.

MYC-AtLCB1 variants transgenic Arabidopsis plants were generated using the Agrobacterium tumefaciens-mediated floral-dip method ${ }^{66}$ and screened by treatment with $15 \mathrm{mg} / \mathrm{L}$ hygromycin and immunoblotting using anti-MYC antibody. Previously generated $M K K 9^{K R}, M K K 9^{D D}, M K K 9^{D D} / m p k 3, M K K 9^{D D} / m p k 6$, mpk3, mpk6 mutant plants were used ${ }^{47}$. The $M K K 9^{D D} / l c b 2 a / l c b 2 b i$ mutant was generated by crossing the $l c b 2 a / l c b 2 b i$ mutant ${ }^{14}$ with $M K K 9^{D D}$ transgenic plants, and homozygous plants were screened.

\section{Vector Construction.}


518 Total RNA was isolated from samples with Trizol reagent (Ambion). Reverse transcription was performed using oligo $\mathrm{dT}(16)$ as a primer and total RNA as the template. M-MLV reverse transcriptase (Promega) was used to reverse transcribe the poly $(\mathrm{A})^{+}$mRNAs.

The coding region of $A t L C B 1$ was PCR amplified using cDNA as template and cloned into the pGEM-T Easy vector. An Nde I site was added before the start codon of AtLCB1. Point mutations were introduced by site-directed mutagenesis PCR. The $A t L C B 1$ native promoter fragment (the $2500 \mathrm{bp}$ before the first ATG of $A t L C B 1$ ) was PCR amplified using genomic DNA as template and cloned into a modified pBlueScript vector with a 4xMYC tag coding sequence. The Nde I and Spe I fragments of each $A t L C B 1$ mutant were then inserted after the MYC tag sequence. The Sal I and Sac I fragments of the native-promoter-MYC-AtLCB1 mutant sequences were cloned into the pCAMBIA 1300 binary vector. The resultant constructs were transformed into A.tumefaciens strain GV3101.

To generate constructs for the split-BiFC assay, the coding sequence of AtLCB1 with a BamH I site added before the start codon and an Xho I site added to replace the stop codon was PCR amplified and cloned into pSPYNE vector to express AtLCB1$\mathrm{YFP}^{\mathrm{NE}}$. The BamH I and Xho I fragments of MPK3 and the BamH I and Sal I fragments of MPK6 from previously used pBlueScript plasmids (Xu et al, 2008, JBC) were cloned into the pSPYCE(MR) vectors to express $\mathrm{YFP}^{\mathrm{CE}}-\mathrm{MPK} 3$ and $\mathrm{YFP}^{\mathrm{CE}}-\mathrm{MPK} 6$. The resultant constructs were transformed into A. tumefaciens strain GV3101.

To generate constructs for the recombinant $\triangle \mathrm{AtLCB} 1$ variant proteins expression, a version of $A t L C B 1$ with the amino acid residues 1 to 60 of the coding sequence deleted was PCR amplified and inserted between the Nde I and Sal I sites of the pET28a(+) vector after the $6 \mathrm{xHis}$ tag coding sequence.

To generate constructs for yeast two-hybrid assays, the coding sequences of AtLCB1, AtLCB2a, AtLCB2b, ORM1, ORM2, TSC10A, TSC10B, SBH1, SBH2, sSSPTa, ssSPTb, MPK3, and MPK6 were PCR amplified. The start codons of all these genes and the stop codons of all the genes except for MPK3 and MPK6 were deleted and Sfi I sites were added to both the $5^{\prime}$ and the $3^{\prime}$ ends of all the genes. Sf $i$ fragments of $M P K 3$ and 
MPK6 were cloned into the pPR3-N vector, and the other genes were cloned into the pBT3-STE vectors.

To generate constructs for Saccharomyces cerevisiae growth assay, sequence including $S C L C B 1$ and its promoter region with a Sac II site added at the 5' end and an Spe I site added at $3^{\prime}$ end was PCR amplified and cloned into the pBlueScript vector. The Sac II and Spe I fragments were cloned into pRS316 to generate the pRS316$S c L C B 1$ vector. The coding region of $A t L C B 2 b$ with an $N d e$ I site added before the start codon was cloned into a modified pBlueScript vector with an HA tag coding sequence. The fragments of the $M Y C-A t L C B 1$ variants and $H A-A t L C B 2$ with BamH I sites added before their start codons and Sal I sites added after their stop codons were PCR amplified. The BamH I and Sal I fragments of the $M Y C-A t L C B 1$ variants were then cloned into the p423GAL vector to generate $p 423 \mathrm{GAL}-M Y C-A t L C B 1$ variant vectors, and $A t L C B 2 b$ was cloned into p425GAL to generate the p425GAL-HA-LCB2b vector. Primers used are listed in Supplemental Table 1.

\section{Generation of $S$. cerevisiae lcb1 $1 \Delta$ mutant and growth rescue by AtLCB1 and} AtLCB2b.

S.cerevisiae cells were grown according to standard procedures. A heterozygous $l c b 1 \Delta$ knockout mutant was generated by transforming a $N A T$ disrupting fragment into a


then transforming the pRS316-ScLCB1 vector into this mutant. The $l c b 1 \Delta+p S 316$ ScLCB1 haploid was obtained by dissecting of the BY4743 diploid. The p425GAL-HA$L C B 2 b$ vector and $p 423 \mathrm{GAL}-M Y C-A t L C B 1$ variant vectors were co-transformed into the $l c b 1 \Delta+p S 316-S c L C B 1$ cells. The resulting mutant strains were grown in the synthetic drop-out medium containing either +GAL/-Ura/-His/-Leu or +GAL/+5FOA/-His/-Leu or +GAL/+5-FOA/-His/-Leu/+PD98059 and their growth phenotypes were observed.

\section{Preparation of recombinant proteins and anti-AtLCB1 antibody.}

Flag-MKK9 ${ }^{\mathrm{DD}}$, His-MPK3, and His-MPK6 proteins were prepared as previously 
described ${ }^{47} \cdot p \mathrm{ET} 28 \mathrm{a}(+)$ constructs for His- $\triangle \mathrm{AtLCB} 1$ variant proteins expression were transformed into Escherichia coli strain BL21. His- $\triangle$ AtLCB1 variant proteins were induced and purified following the same procedures that were used for His-MPK preparation ${ }^{47}$. The purified His- $\triangle \mathrm{AtLCB} 1^{\mathrm{WT}}$ protein was used as an antigen to immunize New Zealand White Rabbit to raise an antibody to AtLCB1 (done by HuaAn Bio-Technology, Hangzhou, China). The antibody was purified using an AminoLink ${ }^{\mathrm{TM}}$ Plus Immobilization Kit (Thermo Fisher Scientific, USA) and used for immunoblot assays.

\section{Immunoblot assays.}

For immunoblot assays, $10 \mu \mathrm{g}$ of total protein, $8.5 \mu \mathrm{g}$ of microsome protein, $0.5 \mu \mathrm{g}$ of recombinant protein per line was separated on 10\% SDS-PAGE gels, and $17 \mu \mathrm{g}$ of microsome protein was separated on $6 \%$ Phos-tag gels. The proteins were transferred to nitrocellulose membranes and detected with the indicated antibodies. The primary antibodies used were anti-His antibody (R\&D Systems), anti-MYC antibody, antiMPK3 antibody, anti-MPK6 antibody, anti- $\alpha$-tubulin antibody (Sigma-Aldrich), and the anti-AtLCB1 antibody described above. Secondary antibodies were horseradish peroxidase-conjugated goat anti-mouse or anti-rabbit antibodies. The membranes were visualized using an enhanced chemiluminescence kit (Roche Applied System).

\section{Kinase assays.}

For in-solution kinase assay, His-MPK3 and His-MPK6 were initially activated with Flag-MKK9 ${ }^{\mathrm{DD}}$ as described previously ${ }^{47}$. Then, $0.5 \mu \mathrm{g}$ of activated His-MPK3 or HisMPK6 was then used to phosphorylate $5 \mu \mathrm{g}$ of His- $\triangle \mathrm{AtLCB} 1$ variant proteins. After the reaction, proteins were separated on $10 \%$ SDS-PAGE, dried, and exposed to X-ray film. In-gel assays were performed as described previously ${ }^{47}$.

\section{Microsome preparation and gel filtration chromatography.}

Microsomes were prepared as described previously with some modification ${ }^{67}$. The extraction buffer was $100 \mathrm{mM}$ HEPES, pH 8.0, containing $5 \mathrm{mM} \mathrm{KCl}, 0.5 \mathrm{mM}$ PMSF, 
$2 \mu \mathrm{g} / \mathrm{mL}$ leupeptide, $2 \mu \mathrm{g} / \mathrm{mL}$ aprotinin, 25\% sucrose, $5 \%$ glycerol, and $1 \times$ PhosSTOP (Roche). Washing buffer was $50 \mathrm{mM}$ HEPES, pH 8.0, containing $0.5 \mathrm{mM}$ PMSF, 2 $\mu \mathrm{g} / \mathrm{ml}$ leupeptide, $2 \mu \mathrm{g} / \mathrm{ml}$ aprotinin, $1 \times$ PhosSTOP (Roche). The isolated microsomes were stored at $-80^{\circ} \mathrm{C}$ until use.

Microsomes were dissolved in $50 \mathrm{mM}$ HEPES, pH 8.0, containing $1 \mathrm{mM}$ EDTA and $0.2 \%$ Triton $\mathrm{X}-100$ and sonicated three times for $10 \mathrm{sec}$ each time. After being centrifuged at $12,000 \times \mathrm{g}$ for $5 \mathrm{~min}$ at $4^{\circ} \mathrm{C}$, the supernatant was loaded onto a gelfiltration column (Superdex 200 10/300 GL) on an GE AKTApurifier ${ }^{\mathrm{TM}} 10$ FPLC system. The eluted fractions were collected and used for SPT activity and AtLCB1 protein assays.

\section{SPT activity assay.}

SPT activity assay was performed as described previously with some modification ${ }^{68}$. Briefly, seedlings were ground in lysis buffer and centrifuged at 2,500 x g for 2 min to remove the cell debris. SPT activity in the supernatants was measured by the HPLCbased detection. SPT activity was calculated based on the formula provided by Rütti et al. (2009) with d17:1(C17 sphingosine, Biomal) as an internal standard. SPT activity in the gel-filtration chromatography fractions was measured as above mentioned.

\section{Sphingolipid long-chain bases analysis.}

Extraction of total sphingolipids, strong alkaline hydrolysis of complex sphingolipids, and o-phthalaldehyde derivatization of released LCBs were performed and detected as described previously ${ }^{15,69,70}$. LCBs in total sphingolipids samples (without alkaline hydrolysis) were directly derivatized and subsequently determined to refer as the free LCBs. o-Phthalaldehyde derivatives of LCBs were analyzed with an Agilent 1100 HPLC apparatus outfitted with a Luna 3u-C18 reverse-phase column (2.0 mm x 150 $\mathrm{mm}$; Phenomenex). LCBs were quantified relative to the internal standard d17:1.

\section{Statistical analysis.}

All data were analyzed using Microsoft Excel and are presented as mean $\pm \mathrm{SD}$. 
Statistical significance among treatments was analyzed by Student's $t$-test. A P value < 0.05 was considered as significant $(* \mathrm{P}<0.05, * * \mathrm{P}<0.01)$.

640

641

642

643

644

645

646

647

648

649

650

651

652

653

654

655

656

657

658

659

$660 \quad 6$

661

662

663

664

665

666

$667 \quad 9$

668

$669 \quad 10$

670

$671 \quad 11$

672

673

674

675

$676 \quad 13$

\section{Accession numbers.}

Detailed sequence data used in this study can be found in The Arabidopsis Information Resource: MKK9 (At1g73500), MPK3 (At3g45640), MPK6 (At2g43790), AtLCB1 (At4g36480), AtLCB2a (At5g23670), AtLCB2b (At3g48780), ORM1 (At1g01230), ORM2 (At5g42000), ssSPTa (At1g06515), ssSPTb (At2g30942), TSC10A (At3g06060), TSC10B (At5g19200), SBH1 (At1g69640), and SBH2 (At1g14290)

\section{REFERENCES}

1 Lingwood, D. \& Simons, K. Lipid Rafts As a Membrane-Organizing Principle. Science 327, 46-50 (2010).

2 Lippincott-Schwartz, J. \& Phair, R. D. Lipids and Cholesterol as Regulators of Traffic in the Endomembrane System. Annu Rev Biophys 39, 559-578 (2010).

3 Deng, X. et al. Ceramide Biogenesis Is Required for Radiation-Induced Apoptosis in the Germ Line of C. elegans. Science 322, 110-115 (2008).

4 Taha, T. A., Mullen, T. D. \& Obeid, L. M. A house divided: Ceramide, sphingosine, and sphingosine-1-phosphate in programmed cell death. BBA-Rev Biomembranes 1758, 2027-2036 (2006).

5 Lopez, P. H. H. \& Schnaar, R. L. Gangliosides in cell recognition and membrane protein regulation. Curr Opin Struc Biol 19, 549-557 (2009).

in Saccharomyces cerevisiae. Prog Lipid Res 45, 447-465 (2006).

7 Ali, U., Li, H., Wang, X. \& Guo, L. Emerging Roles of Sphingolipid Signaling in Plant Response to Biotic and Abiotic Stresses. Mol Plant 11, 1328-1343 (2018).

8 Michaelson, L. V., Napier, J. A., Molino, D. \& Faure, J.-D. Plant sphingolipids: Their importance in cellular organization and adaption. BBA-Mol Cell Biol Lipids 1861, 1329-1335 (2016).

9 Markham, J. E., Lynch, D. V., Napier, J. A., Dunn, T. M. \& Cahoon, E. B. Plant sphingolipids: function follows form. Curr Opin Plant Biol 16, 350-357 (2013).

10 Guo, L. et al. Connections between Sphingosine Kinase and Phospholipase D in the Abscisic Acid Signaling Pathway in Arabidopsis. J Biol Chem 287, 8286-8296 (2012).

1 Bi, F.-C. et al. Loss of Ceramide Kinase in Arabidopsis Impairs Defenses and Promotes Ceramide Accumulation and Mitochondrial $\mathrm{H}_{2} \mathrm{O}_{2}$ Bursts. Plant Cell 26, 3449-3467 (2014).

12 Luttgeharm, K. D. et al. Overexpression of Arabidopsis Ceramide Synthases Differentially Affects Growth, Sphingolipid Metabolism, Programmed Cell Death, and Mycotoxin Resistance. Plant Physiol 169, 1108-1117 (2015).

13 Li, J. et al. Orosomucoid Proteins Interact with the Small Subunit of Serine Palmitoyltransferase 
and Contribute to Sphingolipid Homeostasis and Stress Responses in Arabidopsis. Plant Cell 28, 3038-3051 (2016).

Dietrich, C. R. et al. Loss-of-function mutations and inducible RNAi suppression of Arabidopsis LCB2 genes reveal the critical role of sphingolipids in gametophytic and sporophytic cell viability. Plant J 54, 284-298 (2008).

Chen, M., Han, G., Dietrich, C. R., Dunn, T. M. \& Cahoon, E. B. The Essential Nature of Sphingolipids in Plants as Revealed by the Functional Identification and Characterization of the Arabidopsis LCB1 Subunit of Serine Palmitoyltransferase. Plant Cell 18, 3576-3593 (2006).

6 Chen, M., Markham, J. E., Dietrich, C. R., Jaworski, J. G. \& Cahoon, E. B. Sphingolipid LongChain Base Hydroxylation Is Important for Growth and Regulation of Sphingolipid Content and Composition in Arabidopsis. Plant Cell 20, 1862-1878 (2008).

17 Dutilleul, C. et al. Phytosphingosine-phosphate is a signal for AtMPK6 activation and Arabidopsis response to chilling. New Phytol 194, 181-191 (2012).

de Silva, K., Laska, B., Brown, C., Sederoff, H. W. \& Khodakovskaya, M. Arabidopsis thaliana calcium-dependent lipid-binding protein (AtCLB): a novel repressor of abiotic stress response. $J$ Exp Bot 62, 2679-2689 (2011).

9 Lenarčič, T. et al. Eudicot plant-specific sphingolipids determine host selectivity of microbial NLP cytolysins. Science 358, 1431-1434 (2017).

Hanada, K. Serine palmitoyltransferase, a key enzyme of sphingolipid metabolism. BBA-Mol Cell Biol Lipids 1632, 16-30 (2003).

Hojjati, M. R., Li, Z. \& Jiang, X. Serine palmitoyl-CoA transferase (SPT) deficiency and sphingolipid levels in mice. BBA-Mol Cell Biol Lipids 1737, 44-51 (2005).

Nagiec, M. M., Baltisberger, J. A., Wells, G. B., Lester, R. L. \& Dickson, R. C. The LCB2 gene of Saccharomyces and the related LCB1 gene encode subunits of serine palmitoyltransferase, the initial enzyme in sphingolipid synthesis. Proc Natl Acad Sci. USA 91, 7899-7902 (1994). Han, G. et al. Identification of small subunits of mammalian serine palmitoyltransferase that confer distinct acyl-CoA substrate specificities. Proc Natl Acad Sci. USA 106, 8186-8191 (2009). Gable, K., Slife, H., Bacikova, D., Monaghan, E. \& Dunn, T. M. Tsc3p Is an 80-Amino Acid Protein Associated with Serine Palmitoyltransferase and Required for Optimal Enzyme Activity. J Biol Chem 275, 7597-7603 (2000).

5 Kimberlin, A. N. et al. Arabidopsis 56-Amino Acid Serine Palmitoyltransferase-Interacting Proteins Stimulate Sphingolipid Synthesis, Are Essential, and Affect Mycotoxin Sensitivity. Plant Cell 25, 4627-4639 (2013).

Kimberlin, A. N. et al. ORM Expression Alters Sphingolipid Homeostasis and Differentially Affects Ceramide Synthase Activity. Plant Physiol 172, 889-900 (2016).

27 Han, S., Lone, M. A., Schneiter, R. \& Chang, A. Orm1 and Orm2 are conserved endoplasmic reticulum membrane proteins regulating lipid homeostasis and protein quality control. Proc Natl Acad Sci. USA 107, 5851-5856 (2010).

28 Wang, S., Robinet, P., Smith, J. D. \& Gulshan, K. ORMDL orosomucoid-like proteins are degraded by free-cholesterol-loading-induced autophagy. Proc Natl Acad Sci. USA 112, 37283733 (2015).

Roelants, F. M., Breslow, D. K., Muir, A., Weissman, J. S. \& Thorner, J. Protein kinase Ypk1 phosphorylates regulatory proteins Orm1 and Orm2 to control sphingolipid homeostasis in Saccharomyces cerevisiae. Proc Natl Acad Sci. USA 108, 19222-19227 (2011). 
Hjelmqvist, L. et al. ORMDL proteins are a conserved new family of endoplasmic reticulum membrane proteins. Genome Biol 3, H0027 (2002).

Olsen, J. V. et al. Global, In Vivo, and Site-Specific Phosphorylation Dynamics in Signaling Networks. Cell 127, 635-648 (2006).

Ernst, D. et al. Novel HSAN1 Mutation in Serine Palmitoyltransferase Resides at a Putative Phosphorylation Site That Is Involved in Regulating Substrate Specificity. NeuroMol Med 17, 47-57 (2015). Tyrosine 164 inhibits its activity and promotes cell survival. J Biol Chem (2013).

MAPK-Group. Mitogen-activated protein kinase cascades in plants: a new nomenclature. Trends Plant Sci 7, 301-308 (2002).

Teige, M. et al. The MKK2 pathway mediates cold and salt stress signaling in Arabidopsis. Mol Cell 15, 141-152 (2004).

Takahashi, F. et al. The mitogen-activated protein kinase cascade MKK3-MPK6 is an important part of the jasmonate signal transduction pathway in Arabidopsis. Plant Cell 19, 805-818 (2007). Ren, D., Yang, H. \& Zhang, S. Cell death mediated by MAPK Is associated with hydrogen peroxide production in Arabidopsis. J Biol Chem 277, 559-565 (2002).

Wang, P., Du, Y., Li, Y., Ren, D. \& Song, C. Hydrogen peroxide-mediated activation of MAP kinase 6 modulates nitric oxide biosynthesis and signal transduction in Arabidopsis. Plant Cell 22, 2981-2998 (2010).

Asai, T. et al. MAP kinase signalling cascade in Arabidopsis innate immunity. Nature 415, 977983 (2002).

Wang, Y. et al. A Pseudomonas syringae ADP-ribosyltransferase inhibits Arabidopsis mitogenactivated protein kinase kinases. Plant Cell 22, 2033-2044 (2010).

1 Li, H. et al. MPK3- and MPK6-mediated ICE1 phosphorylation negatively regulates ICE1 stability and freezing tolerance in Arabidopsis. Dev Cell 43, 630-642 (2017).

Li, K. et al. AIK1, a mitogen-activated protein kinase, modulates abscisic acid responses through the MKK5-MPK6 kinase cascade. Plant Physiol 173, 1391-1408 (2017).

Wang, H., Ngwenyama, N., Liu, Y., Walker, J. C. \& Zhang, S. Stomatal development and patterning are regulated by environmentally responsive mitogen-activated protein kinases in Arabidopsis. Plant Cell 19, 63-73 (2007).

Wang, H. et al. Haplo-insufficiency of MPK3 in MPK6 mutant background uncovers a novel function of these two MAPKs in Arabidopsis ovule development. Plant Cell 20, 602-613 (2008). Jia, W. et al. Mitogen-activated protein kinase cascade MKK7-MPK6 plays important roles in plant development and regulates shoot branching by phosphorylating PIN1 in Arabidopsis. PLOS BIOL 14, e1002550 (2016). Liu, Y. \& Zhang, S. Phosphorylation of 1-aminocyclopropane-1-carboxylic acid synthase by MPK6, a stress-responsive mitogen-activated protein kinase, induces ethylene biosynthesis in Arabidopsis. Plant Cell 16, 3386-3399 (2004).

$\mathrm{Xu}$, J. et al. Activation of MAPK kinase 9 induces ethylene and camalexin biosynthesis and enhances sensitivity to salt stress in Arabidopsis. J Biol Chem 283, 26996-27006 (2008).

Yoo, S.-D., Cho, Y.-H., Tena, G., Xiong, Y. \& Sheen, J. Dual control of nuclear EIN3 by bifurcate MAPK cascades in C2H4 signalling. Nature 451, 789-795 (2008). 
thaliana. New Phytol. 203, 1146-1160 (2014).

50 Xin, X. et al. Arabidopsis MKK10-MPK6 mediates red-light-regulated opening of seedling cotyledons through phosphorylation of PIF3. J Exp Bot 69, 423-439 (2018).

51 Saucedo-García, M. et al. MPK6, sphinganine and the LCB2a gene from serine palmitoyltransferase are required in the signaling pathway that mediates cell death induced by long chain bases in Arabidopsis. New Phytol 191, 943-957 (2011).

$2 \mathrm{Su}, \mathrm{T}$. et al. Glutathione-indole-3-acetonitrile is required for camalexin biosynthesis in Arabidopsis thaliana. Plant Cell 23, 364-380 (2011).

$53 \mathrm{Xu}$, J. \& Chua, N.-H. Dehydration stress activates Arabidopsis MPK6 to signal DCP1 phosphorylation. EMBO J 31, 1975-1984 (2012).

54 Galletti, R., Ferrari, S. \& De Lorenzo, G. Arabidopsis MPK3 and MPK6 play different roles in basal and oligogalacturonide- or flagellin-induced resistance against Botrytis cinerea. Plant Physiol 157, 804-814 (2011).

55 Aoyama, T. \& Chua, N.-H. A glucocorticoid-mediated transcriptional induction system in transgenic plants. Plant J 11, 605-612 (1997).

56 Breslow, D. K. et al. Orm family proteins mediate sphingolipid homeostasis. Nature 463, 10481053 (2010).

57 Siow, D. L. \& Wattenberg, B. W. Mammalian ORMDL Proteins Mediate the Feedback Response in Ceramide Biosynthesis. Journal of Biological Chemistry 287, 40198-40204 (2012).

58 Breslow, D. Sphingolipid homeostasis in the endoplasmic reticulum and beyond. Cold Spring Harb Perspect Biol 5, a013326 (2013).

59 Yasuda, S., Nishijima, M. \& Hanada, K. Localization, Topology, and Function of the LCB1 Subunit of Serine Palmitoyltransferase in Mammalian Cells. J Biol Chem 278, 4176-4183 (2003).

60 Meng, X. \& Zhang, S. MAPK cascades in plant disease resistance signaling. Annu Rev Phytopathol 51, 245-266 (2013).

61 Pitzschke, A., Schikora, A. \& Hirt, H. MAPK cascade signalling networks in plant defence. Curr Opin Plant Biol 12, 421-426 (2009).

62 Magnin-Robert, M. et al. Modifications of Sphingolipid Content Affect Tolerance to Hemibiotrophic and Necrotrophic Pathogens by Modulating Plant Defense Responses in Arabidopsis. Plant Physiol 169, 2255-2274 (2015).

63 Shi, L. et al. Involvement of sphingoid bases in mediating reactive oxygen intermediate production and programmed cell death in Arabidopsis. Cell Res 17, 1030-1040 (2007).

Peer, M., Stegmann, M., Mueller, M. J. \& Waller, F. Pseudomonas syringae infection triggers de novo synthesis of phytosphingosine from sphinganine in Arabidopsis thaliana. FEBS Letters 584, 4053-4056 (2010).

Han, G. et al. The Topology of the Lcb1p Subunit of Yeast Serine Palmitoyltransferase. J Biol Chem 279, 53707-53716 (2004).

Clough, S. J. \& Bent, A. F. Floral dip: a simplified method for Agrobacterium-mediated transformation of Arabidopsis thaliana. Plant J. 16, 735-743 (1998).

67 Abas, L. \& Luschnig, C. Maximum yields of microsomal-type membranes from small amounts of plant material without requiring ultracentrifugation. Anal Biochem 401, 217-227 (2010).

68 Rütti, M. F., Richard, S., Penno, A., von Eckardstein, A. \& Hornemann, T. An improved method to determine serine palmitoyltransferase activity. J Lipid Res 50, 1237-1244 (2009). 
69 Morrison, W. R. \& Hay, J. D. Polar lipids in bovine milk. II. Long-chain bases, normal and 2hydroxy fatty acids, and isomeric cis and trans monoenoic fatty acids in the sphingolipids. Biochim Biophys Acta 202, 460-467 (1970).

70 Wright, B. S., Snow, J. W., O’Brien, T. C. \& Lynch, D. V. Synthesis of 4-hydroxysphinganine and characterization of sphinganine hydroxylase activity in corn. Arch Biochem Biophys 415, 184-192 (2003).

\section{Acknowledgements}

We thank Dr. Edgar B. Cahoon in the Department of Biochemistry, University of Nebraska-Lincoln, USA, for providing us with Arabidopsis $l c b 2 a / l c b 2 b R N A i$ seeds. This work was supported by grants from the National Natural Science Foundation of China (grant no. 31670248 and no. 31470351) to DR.

\section{Author contributions}

Y.L., H.C., and D.R. designed the experiments. Y.L., H.C., T.D., X.W., L.M., and K.L. conducted the experiments and collected and analyzed the data; Y.L., H.C., H.L., C.S. and D.R. wrote the paper with contributions from all other authors.

\section{Competing interests}

The authors declare no competing interests.

\section{Supplementary information}

Supplementary Table 1 and Supplementary Figures 1-6. 
a
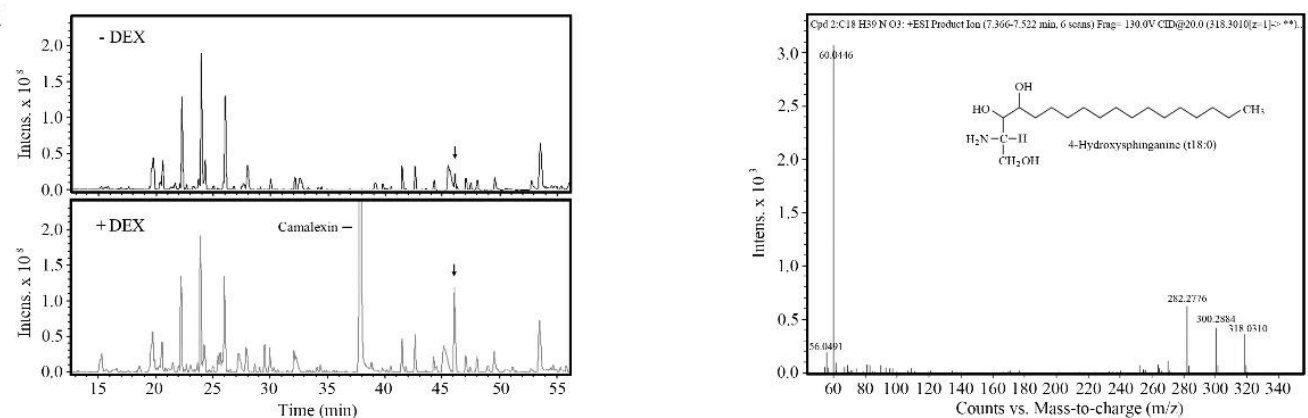

b


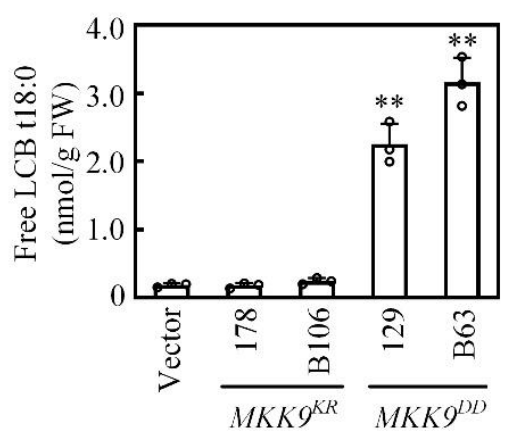

d



Figure 1. MKK9 activates MPK3 and MPK6 to modulate sphingolipid

836 biosynthesis. a, Mass spectral detection of methanol-extracted metabolites from $M K K 9^{D D}$ seedlings before (-DEX) or after (+DEX) MKK9 ${ }^{\mathrm{DD}}$ induction. The arrows

838 indicate the peaks increased significantly after $\mathrm{MKK} 9^{\mathrm{DD}}$ induction except for camalexin 
839 and further analyzed by mass spectrometry. b, Contents of free $\operatorname{LCB}(\mathrm{P})$, ceramide, 840 hydroxyceramide, glucosylceramide, and GIPC complex sphingolipid species 841 characterized by LCB (d18:0, d18:1, t18:0, and t18:1) and fatty acid (16:0-26:1) 842 pairings in $M K K 9^{D D}$ seedlings before (-DEX) or after (+DEX) MKK9 ${ }^{\mathrm{DD}}$ induction. 843 Data represent the means \pm SD of five biological replicates. Asterisks indicate 844 significant difference between $M K K 9^{D D}$ seedlings before (-DEX) and after (+DEX) $845 \mathrm{MKK}^{\mathrm{DD}}$ induction (two-tailed Student's $t$-test, ${ }^{*} P<0.05, * * P<0.01$ ). c, Free LCB $846 \mathrm{t} 18: 0$ contents in $M K K 9^{K R}, M K K 9^{D D}$, and empty vector transgenic seedlings after the 847 transgenes induction. Data represent the means \pm SD of three biological replicates. 848 Asterisks indicate significant differences between $M K K 9^{D D}$ and $M K K 9^{K R}$ or Vector 849 seedlings after DEX treatment (two-tailed Student's $t$-test, $* * P<0.01$ ). d, Time-course 850 analysis of free LCB t18:0 contents in $M K K 9^{D D}, M K K 9^{D D} / m p k 3$, and $M K K 9^{D D} / m p k 6$ 851 seedlings after MKK9 ${ }^{\mathrm{DD}}$ induction (+ DEX). Data represent the means $\pm \mathrm{SD}$ of three 852 biological replicates. Asterisks indicate significant differences between $M K K 9^{D D}$ and $853 M K K 9^{D D} / m p k 3$ or $M K K 9^{D D} / m p k 6$ seedlings after (+DEX) MKK9 ${ }^{\mathrm{DD}}$ induction (two854 tailed Student's $t$-test, $* * P<0.01)$. 


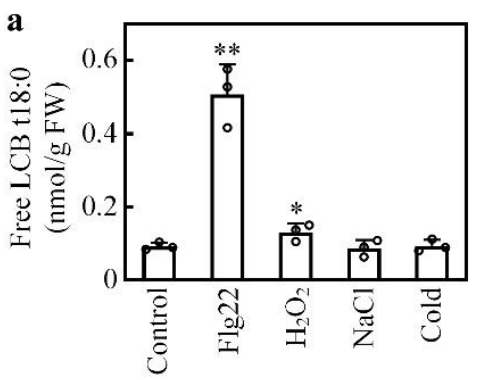

b

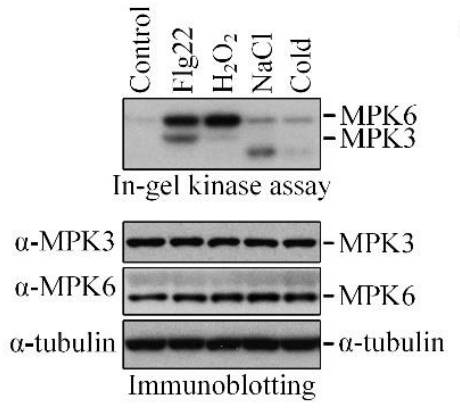

a

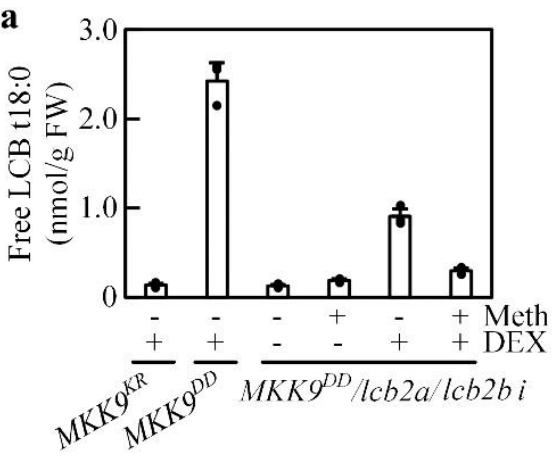

b

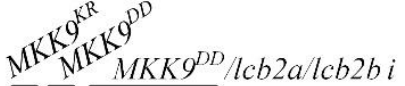

$-\frac{-+-+ \text { Meth }}{-++}$

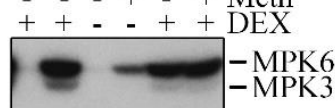

In-gel kinase assay

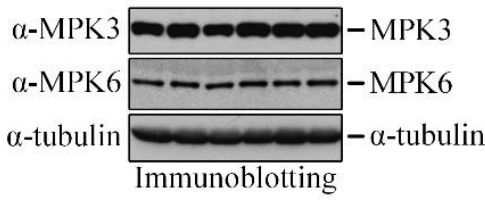

d
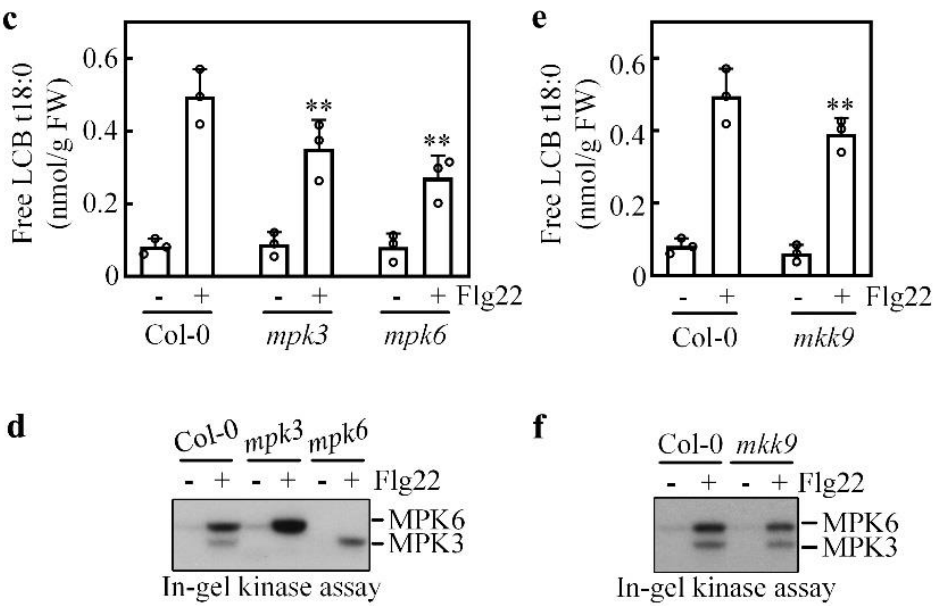

f
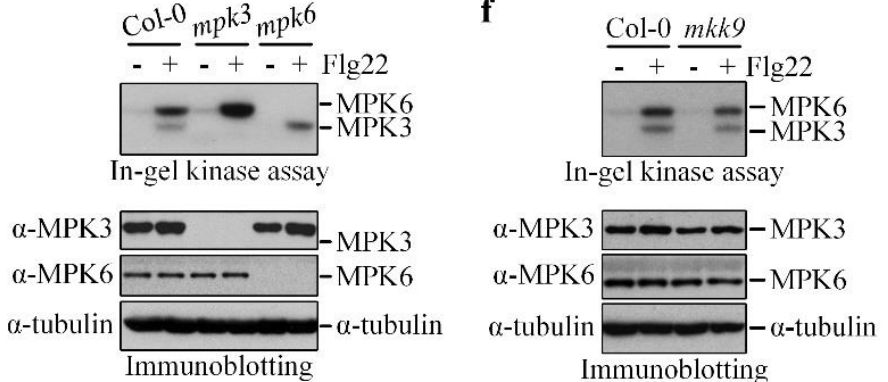

c
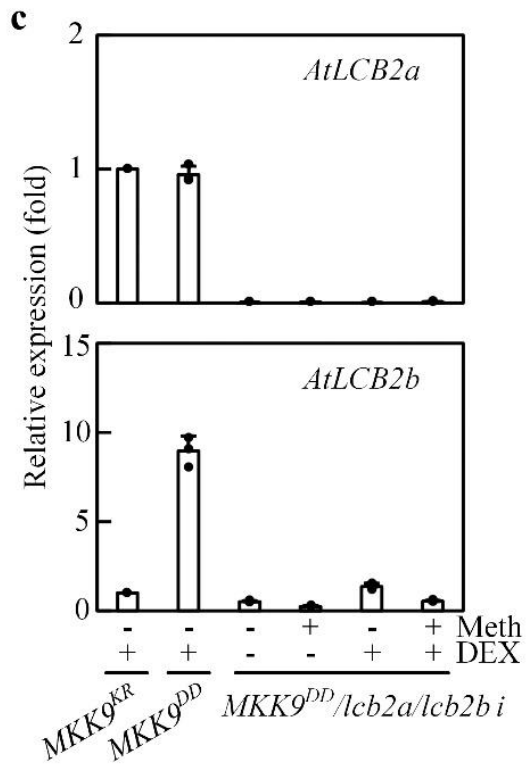

t18:0 accumulation. a, Free LCB t18:0 contents in Col-0 seedlings treated with Flg22, $\mathrm{H}_{2} \mathrm{O}_{2}, \mathrm{NaCl}$, and cold. Free LCB t18:0 content in untreated seedlings was used as control. Data represent the means \pm SD of three biological replicates. Asterisks indicate

863 significant differences between stress-treated and control Col-0 seedlings (two-tailed 864 Student's $t$-test, $\left.{ }^{*} P<0.05,{ }^{*} P<0.01\right) . \mathbf{b}$, In-gel kinase assay and immunoblotting 865 detection of MPK3 and MPK6 in samples from groups of seedlings as in (a). c, Free 
866 LCB t18:0 contents in Col-0, mpk3 and mpk6 seedlings treated with Flg22 (+) or not 867 treated (-). d, In-gel kinase assay and immunoblotting detection of MPK3 and MPK6 868 in samples from groups of seedlings treated as in (c). e, Free LCB t18:0 contents in Col8690 and $m k k 9$ seedlings treated with Flg22 (+) or not treated (-). f, In-gel kinase assay and 870 immunoblotting detection of MPK3 and MPK6 in samples from the same groups of 871 seedlings as in (e).Throughout, -tubulin was used as loading control. In (c) and (e), 872 data represent the means \pm SD of three biological replicates. Asterisks indicate 873 significant differences between Col-0 and $m p k 3$ or $m p k 6$ or $m k k 9$ seedlings treated with 874 Flg22 (two-tailed Student's $t$-test, ${ }^{* *} P<0.01$ ). 


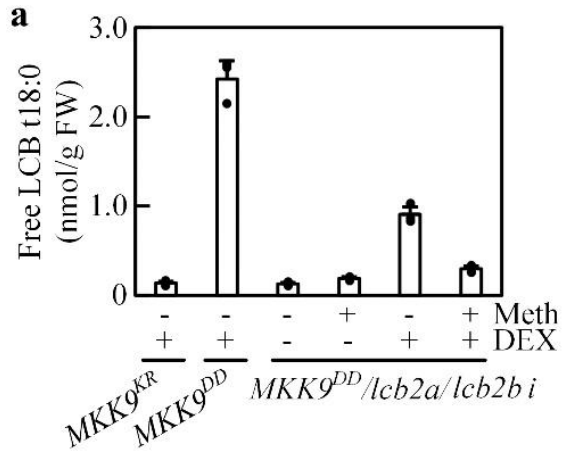

b

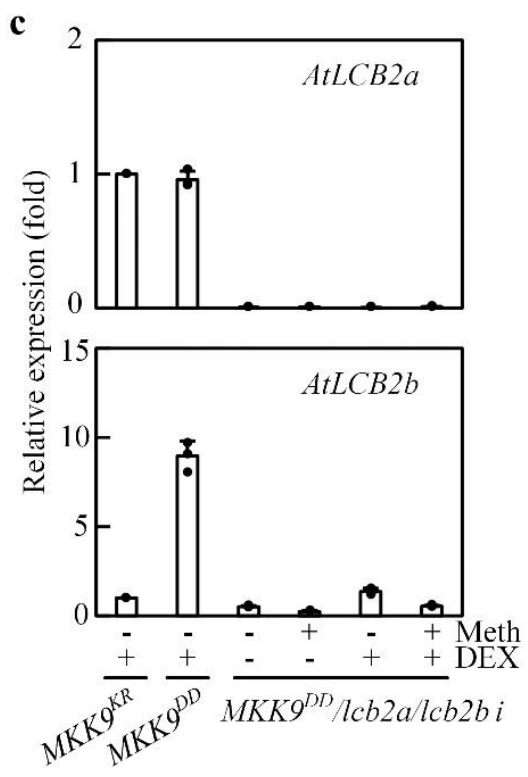

Figure 3. MKK9-MPK3/MPK6-induced LCB t18:0 is from its de novo biosynthesis.

a, Free LCB t18:0 contents in $M K K 9^{K R}, M K K 9^{D D}$, and $M K K 9^{D D} / l c b 2 a / l c b 2 b i$ seedlings with (+) or without (-) transgenes $\left(M K K 9^{K R}, M K K 9^{D D}\right.$, and $\left.A t L C B 2 b R N A i\right)$ induction. b, In-gel kinase assay and immunoblotting detection of MPK3 and MPK6 in samples from seedlings treated as in (a); -tubulin was used as a loading control. c, Relative expression of $A t L C B 2 a$ and $A t L C B 2 b$ in the same groups of seedlings as in (a). Meth, methoxyfenozide (for induction of siRNA expression); DEX, dexamethasone (for induction of $\mathrm{MKK} 9^{\mathrm{DD}}$ expression). Data represent the means $\pm \mathrm{SD}$ of three biological replicates. 


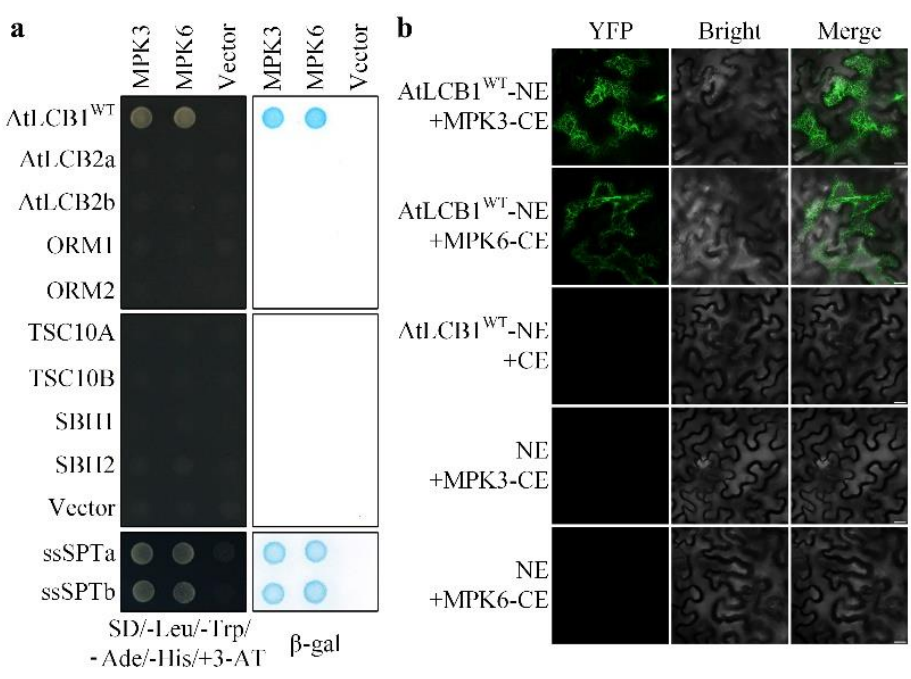

c

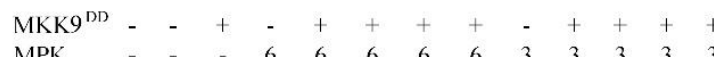
MPK - -

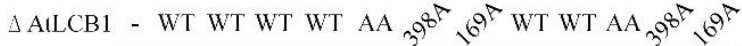

Figure 4. MKK9-MPK3/MPK6 phosphorylates AtLCB1 subunit in SPT. a, Yeast two-hybrid detection of interactions of MPK3 or MPK6 with various enzymes involved in LCB t18:0 biosynthesis and their regulators. $\mathbf{b}, \mathrm{BiFC}$ analysis of the interaction of MPK3 or MPK6 with AtLCB1 using the tobacco leaf epidermal cell transient expression system. c, In vitro phosphorylation of the recombinant AtLCB1 variants by MKK9activated MPK3 and MPK6. d, Phosphorylation of AtLCB1 in Col-0, mpk3, and mpk6 seedlings treated with Flg22 (+) or not treated (-) and in $M K K 9^{D D}, M K K 9^{D D} / m p k 3$, and $M K K 9^{D D} / m p k 6$ seedlings grown with (+) or without (-) MKK9 $9^{\mathrm{DD}}$ induction. Numbers

899 in white show the ratios of phosphorylated to unphosphorylated AtLCB1. DEX, 900 dethamethasone; CIAP, calf intestinal alkaline phosphatase. 

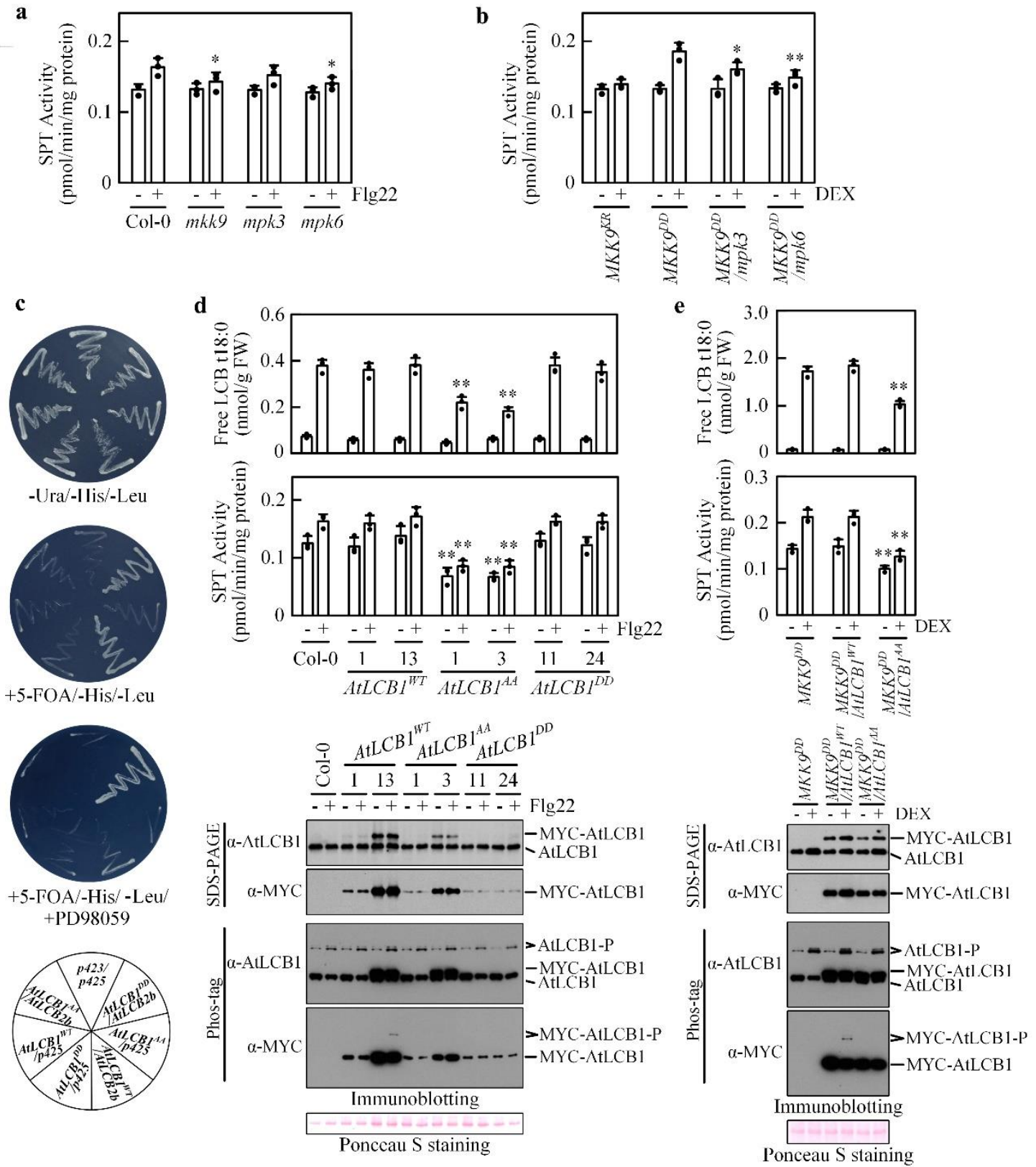

Figure 5. AtLCB1 phosphorylation stimulates SPT activity and free LCB t18:0

biosynthesis. a, SPT activities in Col- $0, m k k 9, m p k 3$, and $m p k 6$ seedlings treated with Flg22 (+) or not treated (-). Data represent the means \pm SD of three biological replicates. Asterisks indicate significant differences between Col-0 and $m p k 3$ or $m p k 6$ or $m k k 9$ seedlings treated with Flg22 (two-tailed Student's $t$-test, $* P<0.05$ ).b, SPT activities in $M K K 9^{K R}, M K K 9^{D D}, M K K 9^{D D} / m p k 3$, and $M K K 9^{D D} / m p k 6$ seedlings grown with (+) or without (-) transgenes induction. Data represent the means \pm SD of three biological replicates. Asterisks indicate significant differences between Col-0 and $m p k 3$ or $m p k 6$ 
912 or $m k k 9$ seedlings treated with Flg22 (two-tailed Student's $t$-test, $* * P<0.01$ ).c, Yeast

$913 \quad l c b 1$ mutant growth rescue by co-expression of $A t L C B 2 b$ with $A t L C B 1$ variant genes.

914 d, SPT activities, free LCB t18:0 contents, and AtLCB1 phosphorylation in AtLCB1

915 variants transgenic seedlings treated with Flg22 (+) or not treated (-). e, SPT activities,

916 free LCB t18:0 contents, and AtLCB1 phosphorylation in $M K K 9^{D D}$,

$917 M K K 9^{D D} / A t L C B 1^{A A}$, and $M K K 9^{D D} / A t L C B 1^{W T}$ seedlings grown with (+) or without (-)

918 MKK9 ${ }^{\mathrm{DD}}$ induction. $* \mathrm{P}<0.05, * * \mathrm{P}<0.01$, Student's $t$-test.

919 
b
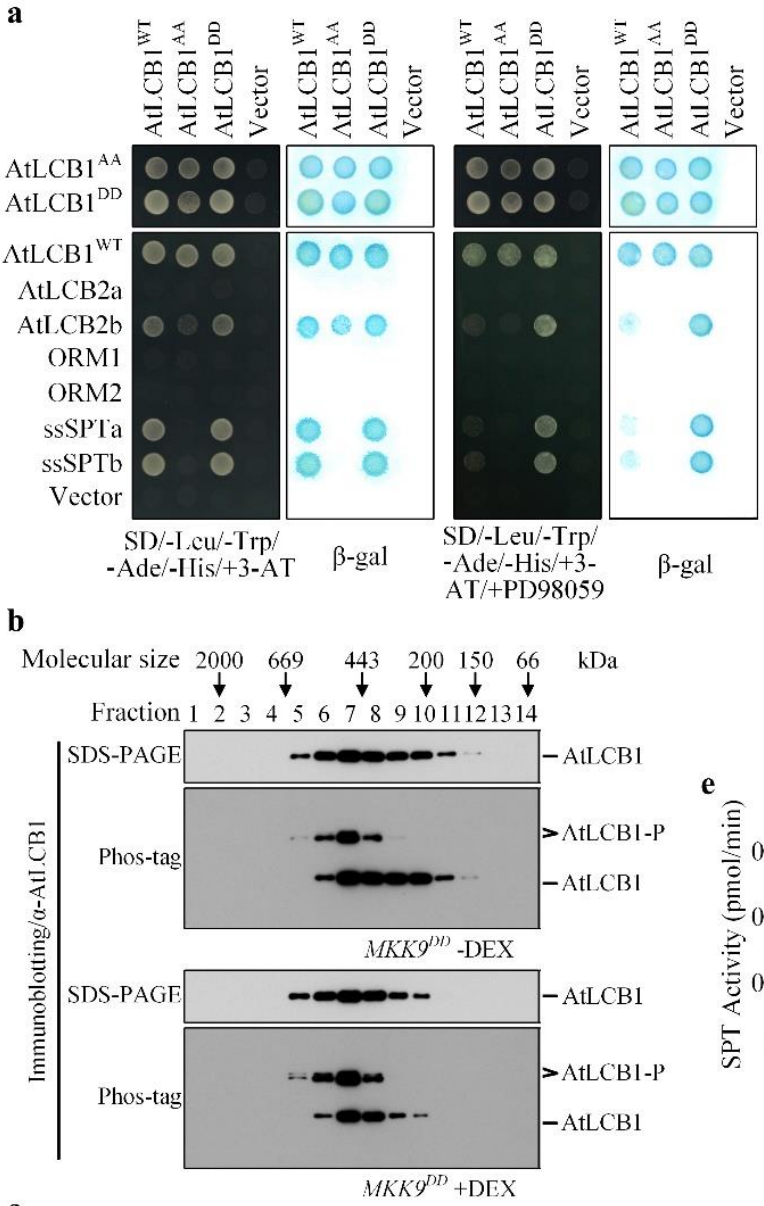

c

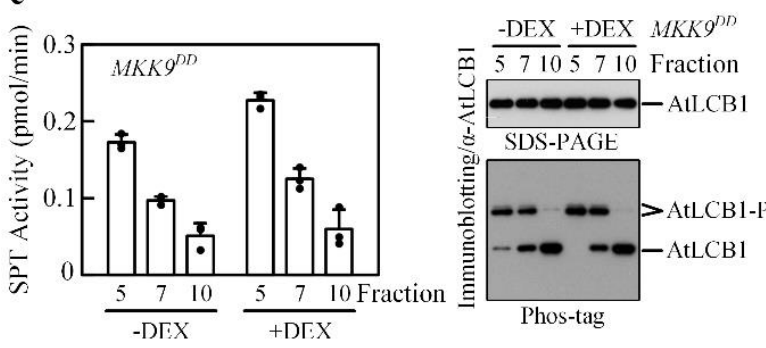

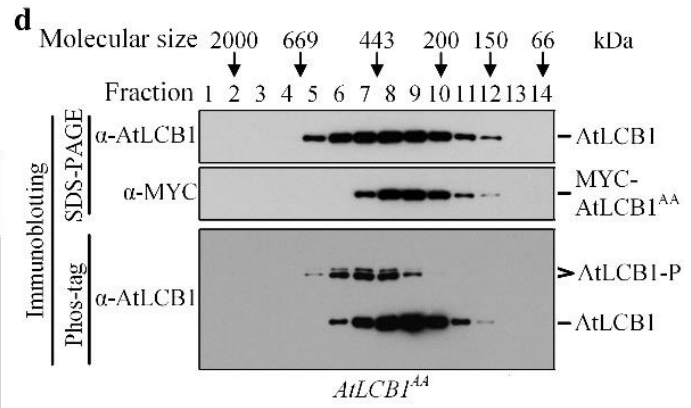
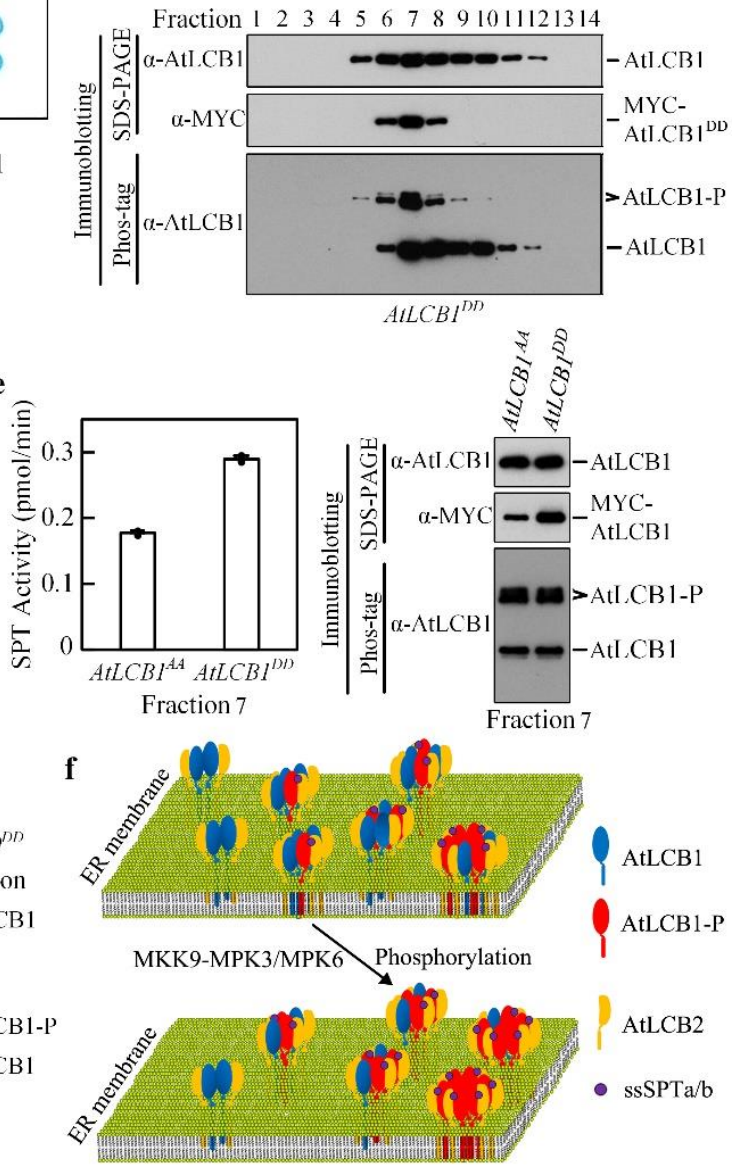

Figure 6. AtLCB1 phosphorylation stimulates higher oligomer and higher activity

SPT formation. a, Yeast two-hybrid detection of interactions between the AtLCB1 variants with each other and with the AtLCB2a, AtLCB2b, ORM1, ORM2, ssSPTa, and ssSPTb in the presence or absence of PD98059. b, Gel-filtration analysis of proteins from $M K K 9^{D D}$ seedlings with or without $\mathrm{MKK} 9^{\mathrm{DD}}$ induction. Fractions (1-14) were collected and analyzed for the presence of AtLCB1 by immunoblotting. c, SPT activities and AtLCB1 proteins in fractions 5, 7, and 10 from (b). Equal amount of AtLCB1 protein for each fraction was used for SPT activity assay. d, Gel filtration analysis of proteins from $A t L C B 1^{A A}$ and $A t L C B 1^{D D}$ seedlings. Fractions were collected 
931 and analyzed as described in (b). e, SPT activities and AtLCB1 proteins in fractions 5,

9327 , and 10 from (d). f, A working model for the regulation of SPT activity by AtLCB1 933 phosphorylation.

934 
936 Phosphorylation of LCB1 subunit of serine palmitoyltransferase stimulates its activity and modulates sphingolipid biosynthesis in Arabidopsis

938

Correspondence to: ren@cau.edu.cn

942

943

This file includes:

$944 \quad$ Table S1

945

Figure S1 to Figure S6

946

947

948 
Table S1. Oligonucleotides used in this study.

\begin{tabular}{|c|c|}
\hline Oligo Name & Oligonucleotide $\left(5^{\prime}-3^{\prime}\right)$ \\
\hline & Primers for genomic PCR screen of mutants \\
\hline \multicolumn{2}{|l|}{ mkk9 (SAIL_060_H06) } \\
\hline mkk9-LP & GGCTTTAGTACGTGAACGTCG \\
\hline mkk9-RP & CCCAAAACTTATGTACACGATTG \\
\hline LB2 for SAIL & GCTTCCTATTATATCTTCCCAAATTACCAATACA \\
\hline \multicolumn{2}{|l|}{ lcb2a-1 (SALK_061472) } \\
\hline lcb2a-1-LP & GAGATTTCTTCCGTCGCTTC \\
\hline lcb2a-1-RP & AGCAGGCTTTCCCACAAACC \\
\hline \multirow[t]{2}{*}{ LB1a for SALK } & TGGTTCACGTAGTGGGCCATCG \\
\hline & Primers for Q-PCR \\
\hline \multicolumn{2}{|l|}{$L C B 1(\mathrm{At} 4 \mathrm{~g} 36480)$} \\
\hline LCB1-Q-LP & CTGTTATGGAAAGGATTGTCAGATA \\
\hline LCB1-Q-RP & CTTTTAGAACTCACAACCAACAATG \\
\hline \multicolumn{2}{|l|}{$L C B 2 a(\mathrm{At5g} 23670)$} \\
\hline LCB2a-Q-LP & GCGACAAAAAGTGGCAGTG \\
\hline LCB2a-Q-RP & TGTTCTATTTTCTTCGGCTCTG \\
\hline \multicolumn{2}{|l|}{$L C B 2 b(\mathrm{At} 3 \mathrm{~g} 48780)$} \\
\hline LCB2b-Q-LP & TCAAACAGAGGGGCACAAAAG \\
\hline LCB2b-Q-RP & CACCACCGCCAAGTTTTCTC \\
\hline \multicolumn{2}{|l|}{ ssSPTa (At1g06515) } \\
\hline ssSPTa-Q-LP & GTCAAGTTCTCTGTCAGAGATTTACCG \\
\hline ssSPTa-Q-RP & TGCTCTACTGTGCGAGAAACAAGACA \\
\hline \multicolumn{2}{|l|}{ ssSPTb (At2g30942) } \\
\hline ssSPTb-Q-LP & GGGTGTTTCCGACCGTCGAATC \\
\hline ssSPTb-Q-RP & ACGATTCCATGGCGTGATGATTAT \\
\hline \multicolumn{2}{|l|}{ ORM1 (At1g01230) } \\
\hline ORM1-Q-LP & GCTTATTCTCTTCTTCGGTTGG \\
\hline ORM1-Q-RP & GAGTTCCTTTCATCCAGTGGAAG \\
\hline \multicolumn{2}{|l|}{ ORM2 (At5g42000) } \\
\hline ORM2-Q-LP & AGATTGACTTGGTGGGAACAGAT \\
\hline ORM2-Q-RP & GTTGAGAAAGAGCATCGGGTG \\
\hline \multicolumn{2}{|l|}{ TSC10A (At3g06060) } \\
\hline TSC10A-Q-LP & ACCGCAGGGCCTATAAGATT \\
\hline TSC10A-Q-RP & GGCTGTCATTTGGTTTTGG \\
\hline \multicolumn{2}{|l|}{ TSC10B (At5g19200) } \\
\hline TSC10B-Q-LP & GGTTTGAACAAGAACTGAAGAAG \\
\hline TSC10B-Q-RP & CTTCATTGGTTTTCATTGAGC \\
\hline \multicolumn{2}{|l|}{ SBH1 (At1g69640) } \\
\hline SBH1-Q-LP & AGATTTTGGAGTTTGGATATATGTTATGTA \\
\hline SBH1-Q-RP & CCAAGCTAAATTCGCTCTTGAG \\
\hline SBH2 (At1g14290) & \\
\hline
\end{tabular}




\begin{tabular}{|c|c|}
\hline SBH2-Q-LP & CTTTCTTTCTTTTGGGGCTTCT \\
\hline SBH2-Q-RP & TACACAGAAACATATGCCCGC \\
\hline \multicolumn{2}{|c|}{$M K K 9$ (Atlg73500) } \\
\hline MKK9-Q-LP & CGACGTTGATGTGCGCGGTGTGT \\
\hline MKK9-Q-RP & CAAGAAGCTGCGGCGCCGTCCA \\
\hline \multicolumn{2}{|c|}{ UBQ5 (At3g62250) } \\
\hline UBQ5-Q-LP & СTCCTTCTTTCTGGTAAACGT \\
\hline \multirow[t]{2}{*}{ UBQ5-Q-RP } & GGTGCTAAGAAGAGGAAGAAT \\
\hline & Primers for vectors construct \\
\hline MPK3 Y2H F & GGCCATTACGGCCAACACCGGCGGTGGCCAATACAC \\
\hline MPK3 Y2H R & GGCCGAGGCGGCCCTAACCGTATGTTGGATTGAGTGCT \\
\hline MPK6 Y2H F & GGCCATTACGGCCGACGGTGGTTCAGGTCAACCGGC \\
\hline MPK6 Y2H R & GGCCGAGGCGGCCCTATTGCTGATATTCTGGATTGAAAG \\
\hline LCB1 Y2H F & GGCCATTACGGCCGCTTCGAATCTCGTGGAAATGTT \\
\hline LCB1 Y2H R & GGCCGAGGCGGCCCCGGACTTGAGTAGAAGCTCTGAAGCAAGT \\
\hline LCB2a Y2H F & GGCCATTACGGCCATAACGATTCCTTATTTAACCGCTGT \\
\hline LCB2a Y2H R & GGCCGAGGCGGCCCCATCCAGCTTGATGTCGTTTTTCGACTGTT \\
\hline LCB2b Y2H F & GGCCATTACGGCCATTACGATCCCATACCTTACCGCTGT \\
\hline LCB2b Y2H R & GGCCGAGGCGGCCCCATCCAATTTGATGCCATTTTTCTCTAC \\
\hline ORM1 Y2H F & GGCCATTACGGCCGCGAATCTGTATGTGAAAGCGGTT \\
\hline ORM1 Y2H R & GGCCGAGGCGGCCCCTTTATCACCATTGATACCAAAGATGCGT \\
\hline ORM2 Y2H F & GGCCATTACGGCCTACGTAAGAGCACTTCCGACGAC \\
\hline ORM2 Y2H R & GGCCGAGGCGGCCCCTTGGTCTCCATTGATTCCAAATATGC \\
\hline TSC10A Y2H R & GGCCGAGGCGGCCCCTTTGGTTTTGGTTTTGCTCCATTTTTCA \\
\hline TSC10B Y2H F & GGCCATTACGGCCGCGGCAATTTTTTCTCTCTTCCTT \\
\hline TSC10B Y2H R & $\begin{array}{l}\text { GGCCGAGGCGGCCCCAGCTAACTTACTATTTACTTCTTTTTTATT } \\
\text { TCTTTGGC }\end{array}$ \\
\hline SBH1 Y2H F & GGCCATTACGGCCATGATGGGTTTTGCTGTATCGGATG \\
\hline SBH1 Y2H R & GGCCGAGGCGGCCCCATCGTCTTTGAATTCTTTAGTCGGGCG \\
\hline $\mathrm{SBH} 2 \mathrm{Y} 2 \mathrm{H} \mathrm{F}$ & GGCCATTACGGCCATGAGTTTCGTGATTTCAGATGAATTTCT \\
\hline $\mathrm{SBH} 2 \mathrm{Y} 2 \mathrm{H} \mathrm{R}$ & GGCCGAGGCGGCCCCCTCATCTTTGGATACTTTGATTGGCC \\
\hline ssSPTa Y2H F & GGCCATTACGGCCAACTGGGTTCAACGC \\
\hline ssSPTa Y2H R & GGCCGAGGCGGCCCCTGTCAAATGCCTCTGG \\
\hline ssSPTb Y2H F & GGCCATTACGGCCAACTGGGTTCAACGAAA \\
\hline ssSPTb Y2H R & GGCCGAGGCGGCCCCTGAAAGATGCCTCT \\
\hline LCB1 BiFC F & GGATCCATGGCTTCGAATCTCGTGGAAATGTT \\
\hline LCB1 BiFC R & CTCGAGGGACTTGAGTAGAAGCTCTGAAGCAAGT \\
\hline LCB1 Prom-LP & GGTACCGTCGACCTGGTAAGGGCTGGATTG \\
\hline LCB1 Prom-RP & CCATGGCTCGAGTGCTAATTTGCTTTAATAA \\
\hline LCB1 CDS-LP & CATATGGCTTCGAATCTCGTGGA \\
\hline LCB1 CDS-RP & TCAGGACTTGAGTAGAAGCTCTGAAG \\
\hline LCB2b CDS-LP & CATATGATTACGATCCCATACCTTACCG \\
\hline LCB2b CDS-RP & TTAATCCAATTTGATGCCATTTTTCT \\
\hline LCB1-169A-LP & TCCAAATTTTTGGGTGCTCCTGATTCAATCCTT \\
\hline
\end{tabular}




\begin{tabular}{ll}
\hline LCB1-169A-RP & AAGGATTGAATCAGGAGCACCCAAAAATTTGGA \\
LCB1-398A-LP & ACAAGCAACCGGGAAGCACCTATTGTTTTCTTA \\
LCB1-398A-RP & TAAGAAAACAATAGGTGCTTCCCGGTTGCTTGT \\
LCB1-169D-LP & TCCAAATTTTTGGGTGATCCTGATTCAATCCTT \\
LCB1-169D-RP & AAGGATTGAATCAGGATCACCCAAAAATTTGGA \\
LCB1-398D-LP & ACAAGCAACCGGGAAGATCCTATTGTTTTCTTA \\
LCB1-398D-RP & TAAGAAAACAATAGGATCTTCCCGGTTGCTTGT \\
ALCB1 CDS-LP & CATATGAAGCGACCATTGACCGAGCAGGA \\
ALCB1 CDS-RP & TCAGGACTTGAGTAGAAGCTCTGAAG \\
Sac II-ScLCB1 F & TCCCCGCGGTGTACTATTTCCATGAACCCAC \\
ScLCB1-SpeI R & GGACTAGTCCTTGTTTGATGTTTGCTGTG \\
ScLCB1-NAT F & TATCCTTTTTTCTTCCTTCCCACCCAAAAAAAAAAAGCACGGAT \\
& CCCCGGGTTAATTAA \\
ScLCB1-NAT R & ATATATATATGTGCGTGTGCATATACTGGCTTTCTATTTGAATTCG \\
& AGCTCGTTTAAAC \\
\hline
\end{tabular}

950

951 
a
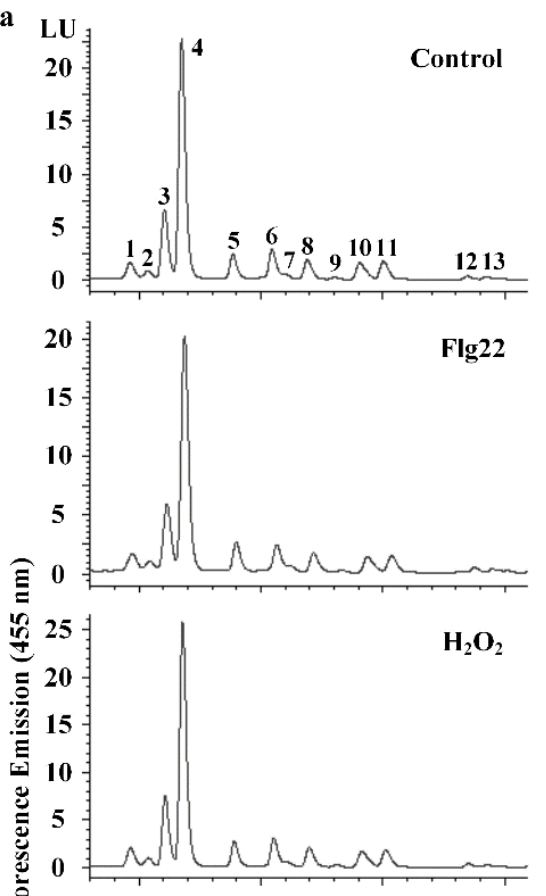

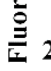
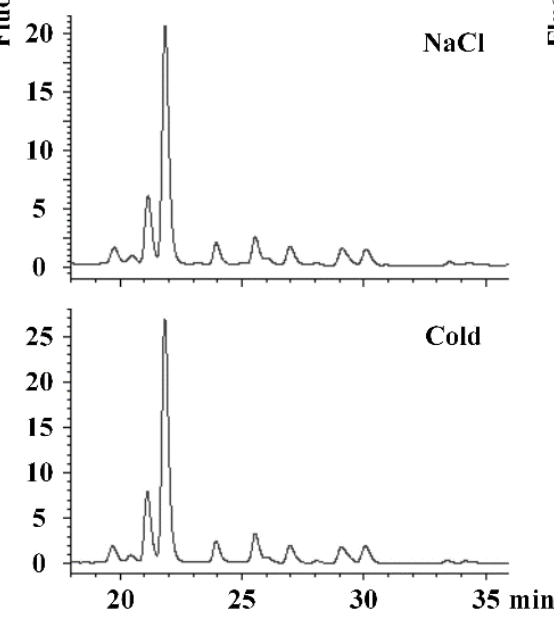



5
4
3
2
1
玉0 0

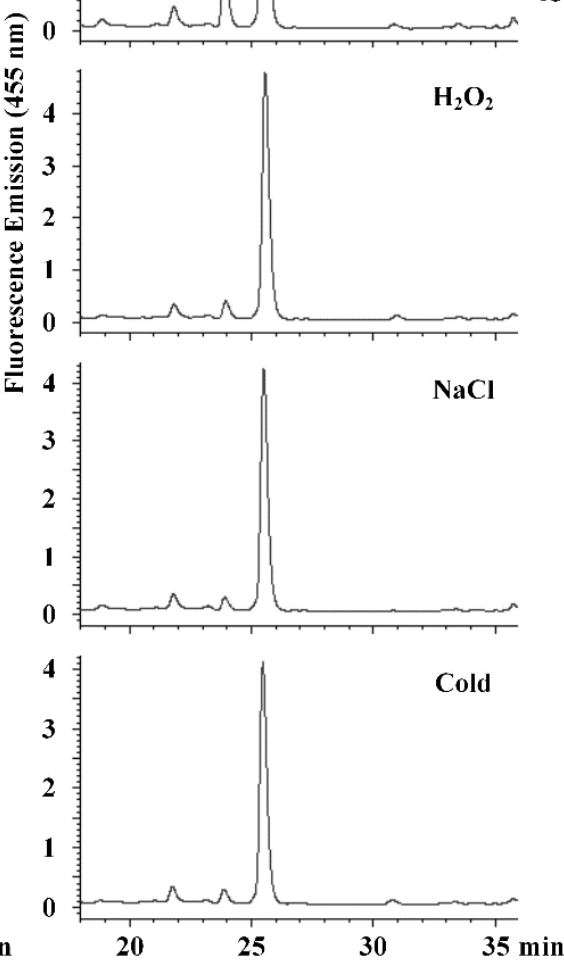

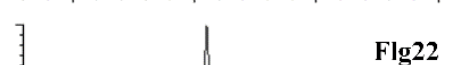

10. d18:1 (8E)

11. 1,4-anhydro-t18: 1 (8E)

12. d18:0

13. 1,4-anhydro-t18:0
1. t18:1 (8Z)-GIc

2. t18: 1 (8E)-GIc

d18:1 (8Z)-Glc

8. d18:1 (8E)-Glc

d18:1 $(8 Z)$

Figure S1. HPLC profiles of total and free LCBs in Col-0 seedlings treated with

$\mathrm{H}_{2} \mathrm{O}_{2}, \mathrm{NaCl}$, and cold. HPLC profiles of total LCBs from untreated Col-0 seedlings was 
$\mathbf{a}$

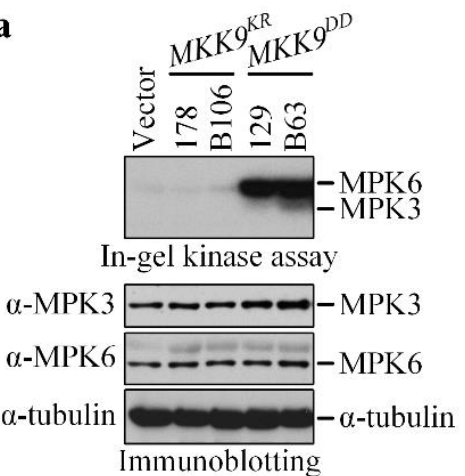



Figure S2. Activation of MPK3 and MPK6 in MKK9 variant transgenic and cross seedlings after DEX treatment. a, In-gel kinase assay and immunoblotting detection of MPK3 and MPK6 in samples from the same group of seedlings as in Figure 1c. b, In-gel kinase assay and immunoblotting detection of MPK3 and MPK6 protein in samples from the same group of seedlings as in Figure 1d. $\alpha$-tubulin was used as a loading control. DEX, dexamethasone (for induction of $\mathrm{MKK} 9^{\mathrm{DD}}$ expression). 

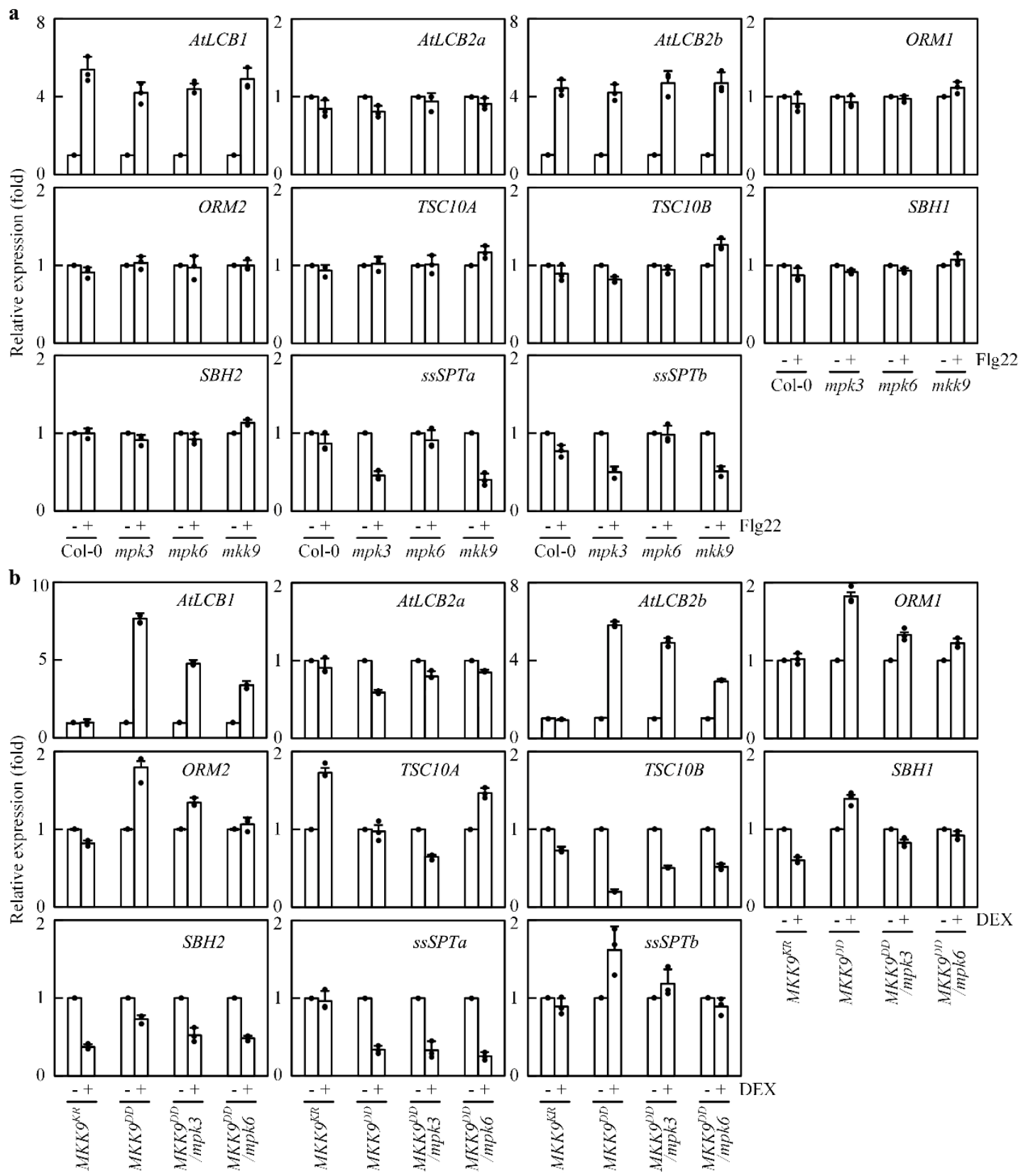

Figure S3. Transcription regulation of genes in LCB t18:0 de novo biosynthetic pathway. a, Relative expression of genes in Col-0, $m k k 9$, $m p k 3$, and $m p k 6$ seedlings

973 treated with Flg22 (+) or not treated (-). b, Relative expression of genes in $M K K 9^{K R}$, $974 M K K 9^{D D}, M K K 9^{D D} / m p k 3$, and $M K K 9^{D D} / m p k 6$ seedlings grown with (+) or without (-) 975 the transgenes induction. DEX, dexamethasone (for induction of MKK9 ${ }^{\mathrm{DD}}$ expression). 

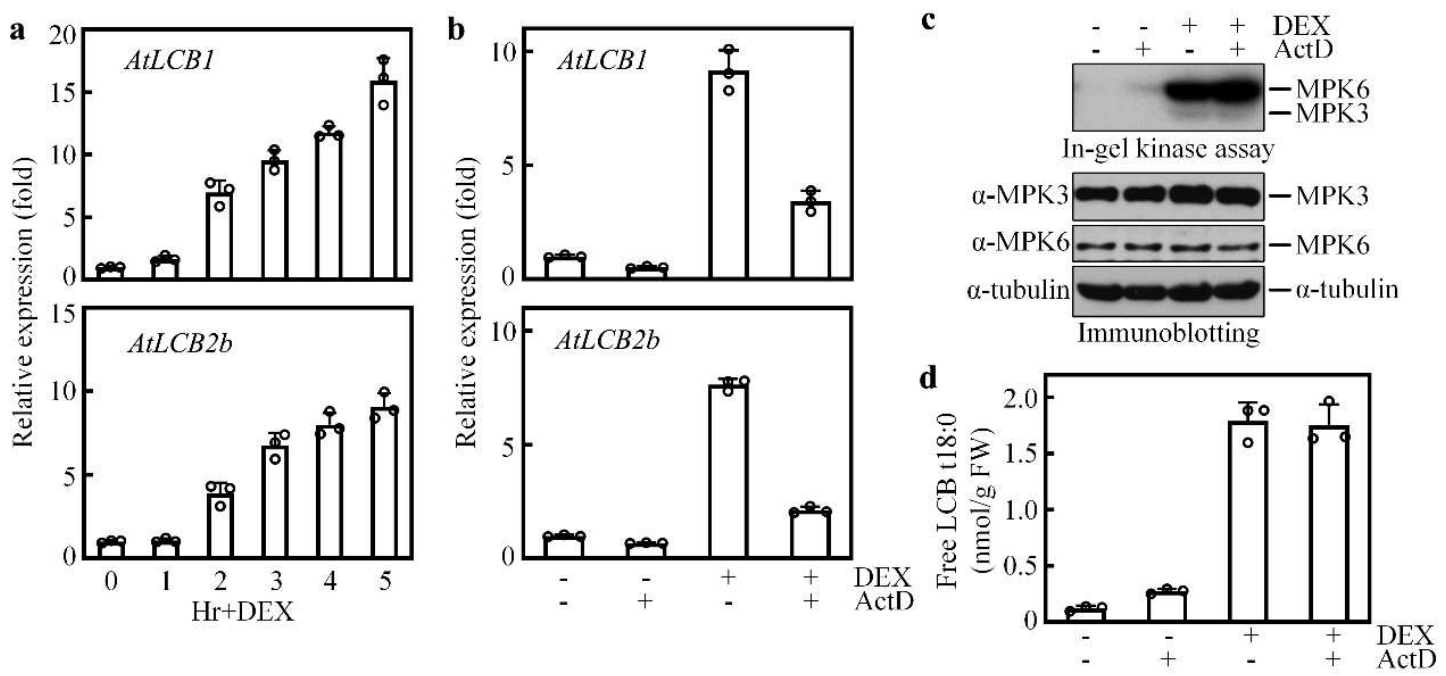

Figure S4. Inhibition of MKK9-MPK3/MPK6-induced transcription of AtLCB1 and $A t L C B 2 b$ does not alter the elevated LCB t18:0 accumulation. a, Relative expression of $A t L C B 1$ and $A t L C B 2 b$ in $M K K 9^{D D}$ seedling after $\mathrm{MKK} 9^{\mathrm{DD}}$ induction for the indicated times. b, Relative expression of $A t L C B 1$ and $A t L C B 2 b$ in $M K K 9^{D D}$ seedling 3 hours after MKK9 ${ }^{\mathrm{DD}}$ induction in the presence or absence of ActD. c, In-gel kinase assay and immunoblotting detection of MPK3 and MPK6 in samples from groups of seedlings as in (b). $\alpha$-tubulin was used as a loading control. d, Free LCB t18:0 contents in samples from groups of seedlings as in (b).ActD treatment was started 1 hour after DEX addition. ActD, actinomycin D (for inhibition of gene's transcription). DEX, dexamethasone (for induction of $\mathrm{MKK} 9^{\mathrm{DD}}$ expression). 


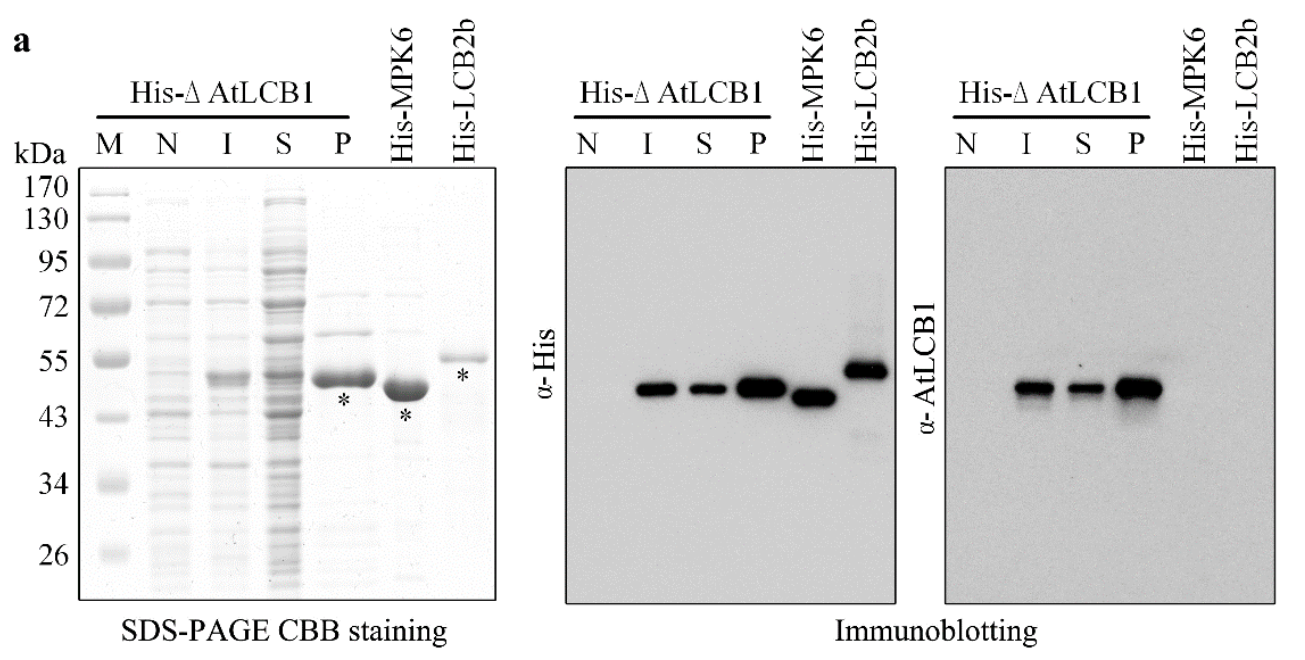

b

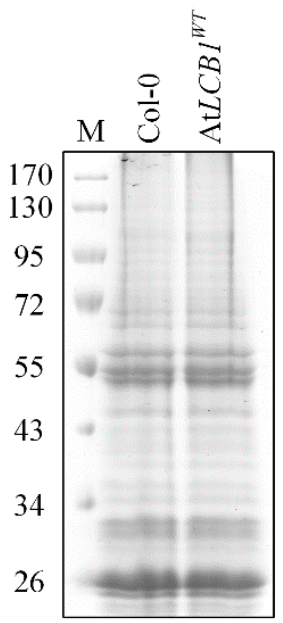

990

SDS-PAGE CBB staining

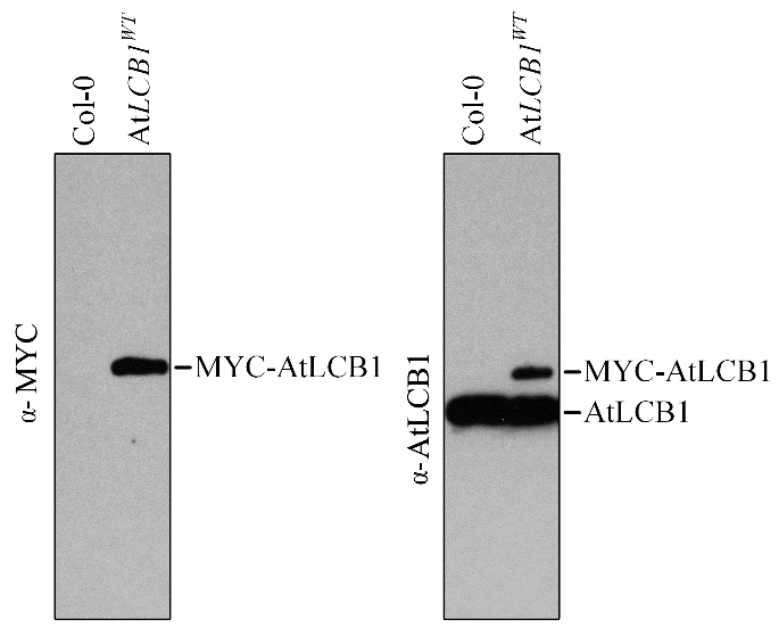

Immunoblotting

991

Figure S5. Detection of specificity of the generated AtLCB1 antibody. a, SDS-

PAGE separation of the recombinant His- $\triangle \mathrm{AtLCB}$, and immunoblotting detection with anti-His and anti-AtLCB1 antibodies. His-MPK6 and His-AtLCB2 were used as controls. M, molecular size marker. N, total proteins in non-induced E. coli cells lysate. I, total proteins in induced $E$. coli cells lysate. S, soluble proteins in induced $E$. coli cell lysate. $\mathrm{P}$, recombinant His- $\triangle \mathrm{AtLCB} 1$ protein purified with a $\mathrm{Ni}^{2+}$-Chelating Sepharose Fast Flow column. Asterisks indicated the purified His- $\triangle$ AtLCB1, His-MPK6, and HisAtLCB2 proteins, respectively. b, SDS-PAGE separation of proteins from microsomes in Col-0 and AtLCB1 ${ }^{W T}$ seedlings, and immunoblotting detection of AtLCB1 with antiMYC and anti-AtLCB1 antibodies. 


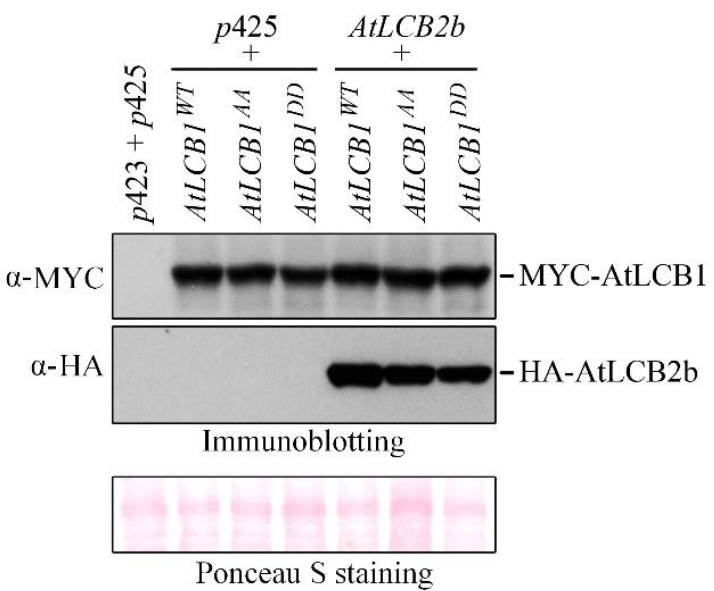

1005 Figure S6. The expression of AtLCB1 variant and AtLCB2b proteins in yeast cells.

1006 Proteins were extracted from the same samples as in Figure 5c and detected by 1007 immunoblot using anti-MYC and anti-HA antibodies. 
Figures

a
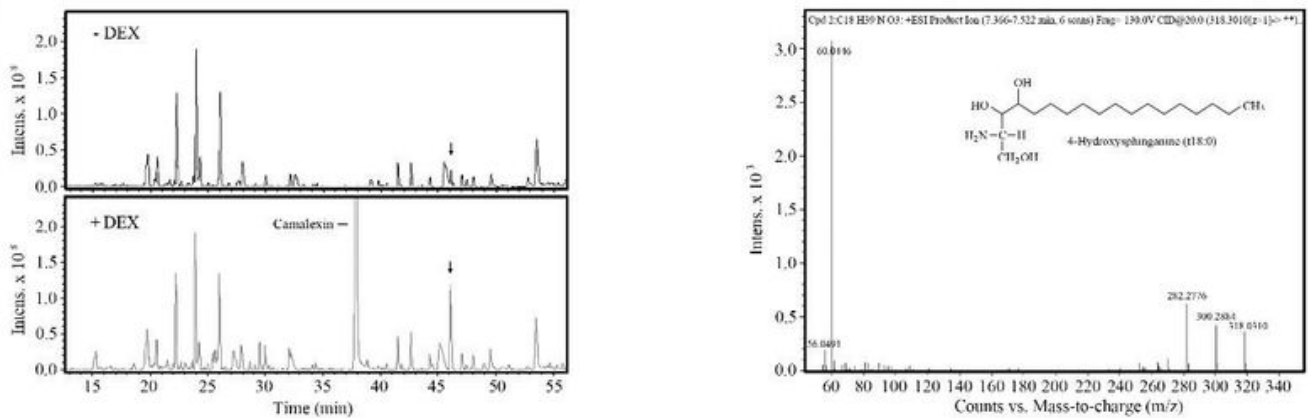

b
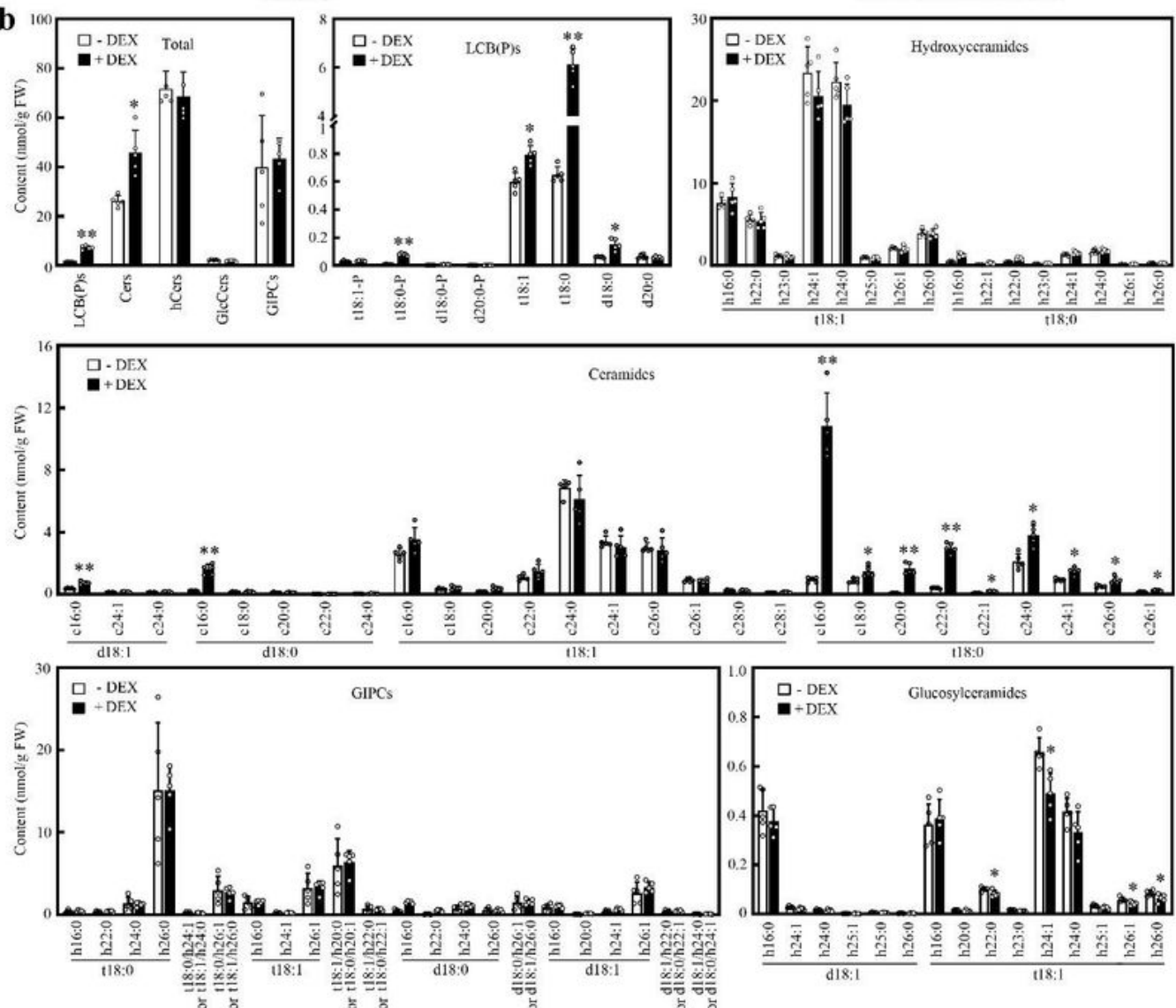

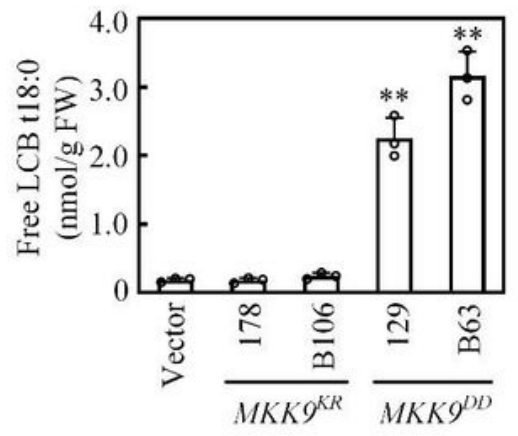

d

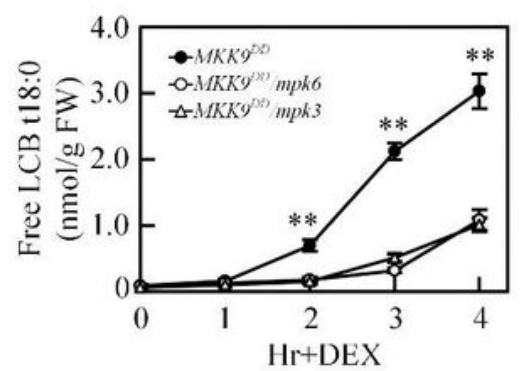

Figure 1

MKK9 activates MPK3 and MPK6 to modulate sphingolipid biosynthesis. a, Mass spectral detection of methanol-extracted metabolites from MKK9DD seedlings before (-DEX) or after (+DEX) MKK9DD induction. The arrowsindicate the peaks increased significantly after MKK9DD induction except for 
camalexinand further analyzed by mass spectrometry. b, Contents of free $\operatorname{LCB}(P)$, ceramide,hydroxyceramide, glucosylceramide, and GIPC complex sphingolipid species characterized by LCB (d18:0, d18:1, t18:0, and t18:1) and fatty acid (16:0-26:1) pairings in MKK9DD seedlings before (DEX) or after (+DEX) MKK9DD induction. Data represent the means $\pm S D$ of five biological replicates. Asterisks indicate significant difference between MKK9DD seedlings before (-DEX) and after (+DEX) 8MKK9DD induction (two-tailed Student's t-test, ${ }^{*} P<0.05$, $* * P<0.01$ ). c, Free LCB t18:0 contents in MKK9KR, MKK9DD, and empty vector transgenic seedlings after the transgenes induction. Data represent the means $\pm S D$ of three biological replicates. Asterisks indicate significant differences between MKK9DD and MKK9KR or Vector seedlings after DEX treatment (two-tailed Student's t-test, ${ }^{*} P<0.01$ ). $d$, Timecourse analysis of free LCB t18:0 contents in MKK9DD, MKK9DD/mpk3, and MKK9DD/mpk6 seedlings after MKK9DD induction (+ DEX). Data represent the means $\pm S D$ of three biological replicates. Asterisks indicate significant differences between MKK9DD and MKK9DD/mpk3 or MKK9DD/mpk6 seedlings after (+DEX) MKK9DD induction (two-tailed Student's t-test, ${ }^{\star * P} \mathrm{P}<0.01$ ). 

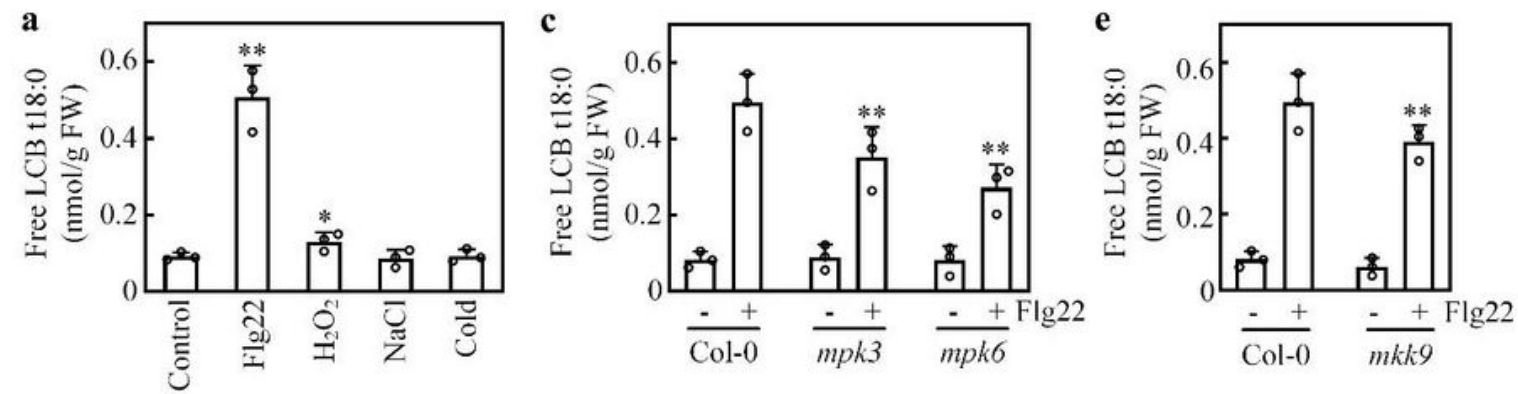

b

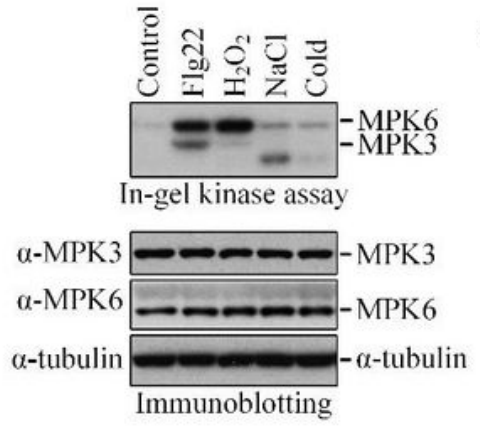

d
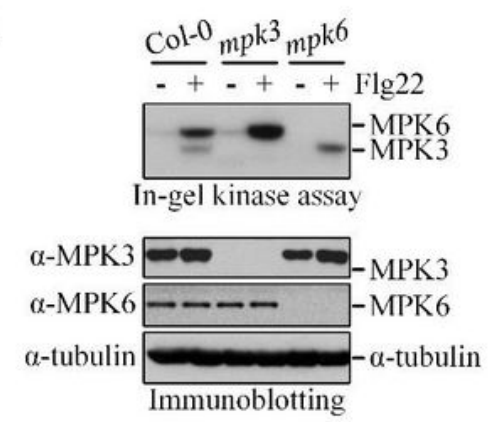

f
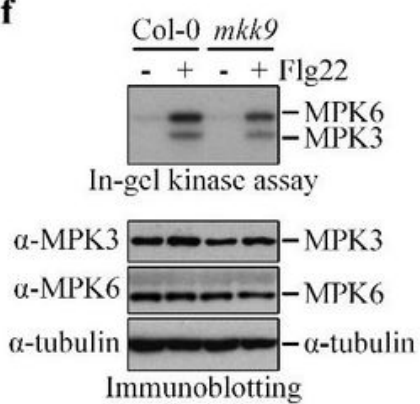

a

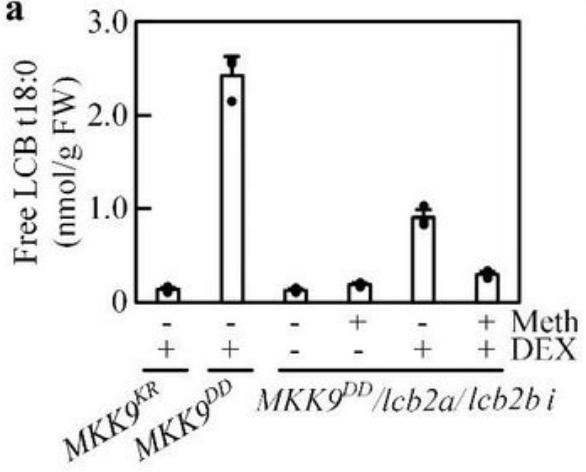

b
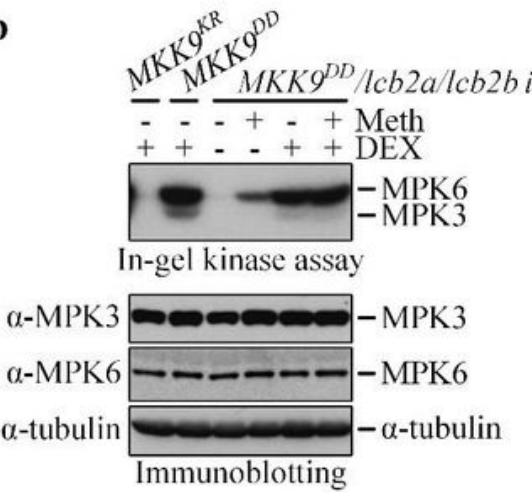

c

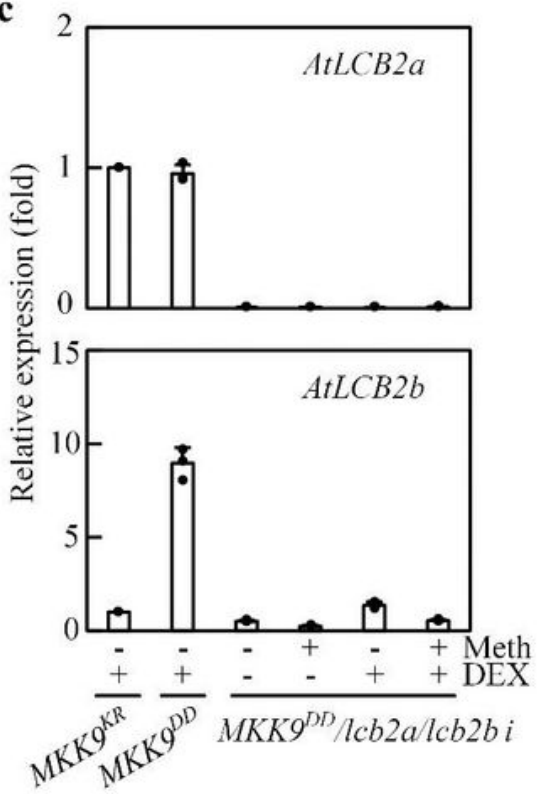

\section{Figure 2}

Flg22 treatment activates MKK9-MPK3/MPK6 and induces free LCB t18:0 accumulation. a, Free LCB t18:0 contents in Col-0 seedlings treated with Flg22, $\mathrm{H} 2 \mathrm{O} 2, \mathrm{NaCl}$, and cold. Free LCB t18:0 content in untreated seedlings was used as control. Data represent the means $\pm S D$ of three biological replicates. Asterisks indicate significant differences between stress-treated and control Col-0 seedlings (two-tailed Student's t-test, $\left.{ }^{*} P<0.05,{ }^{*} \mathrm{P}<0.01\right)$. b, In-gel kinase assay and immunoblotting detection of MPK3 and 
MPK6 in samples from groups of seedlings as in (a). c, Free LCB t18:0 contents in Col-0, mpk3 and mpk6 seedlings treated with Flg22 (+) or not treated (-). d, In-gel kinase assay and immunoblotting detection of MPK3 and MPK6 in samples from groups of seedlings treated as in (c). e, Free LCB t18:0 contents in Col0 and mkk9 seedlings treated with Flg22 (+) or not treated (-). f, In-gel kinase assay and immunoblotting detection of MPK3 and MPK6 in samples from the same groups of seedlings as in (e).Throughout, tubulin was used as loading control. In (c) and (e), data represent the means $\pm S D$ of three biological replicates. Asterisks indicate significant differences between Col-0 and mpk3 or mpk6 or mkk9 seedlings treated with 873 Flg22 (two-tailed Student's t-test, ${ }^{*} \mathrm{P}<0.01$ ).

a

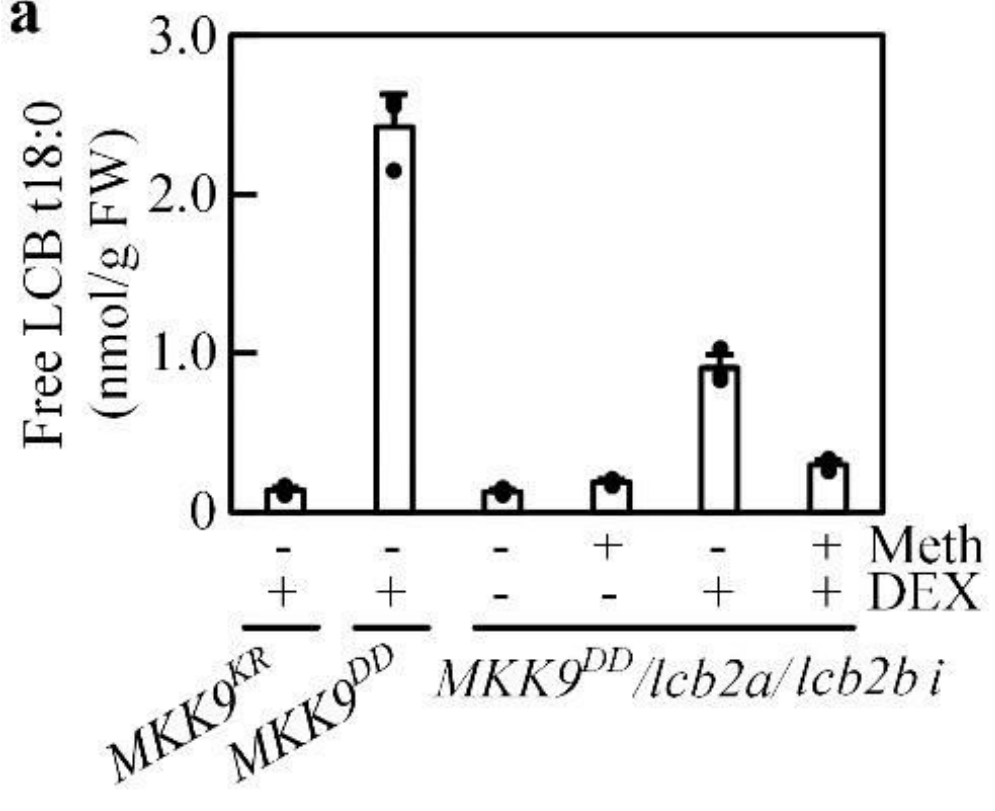

b

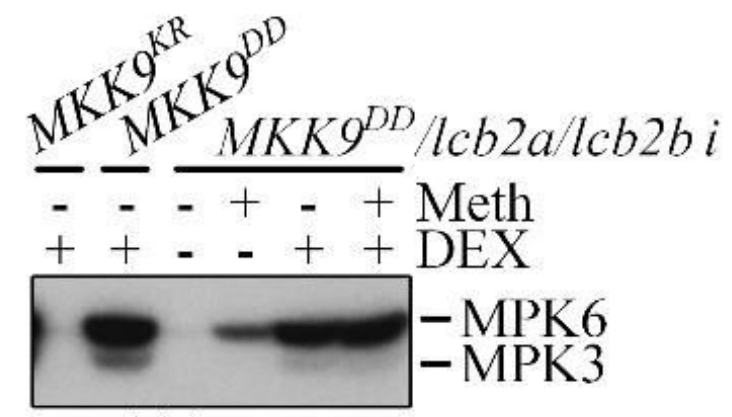

In-gel kinase assay

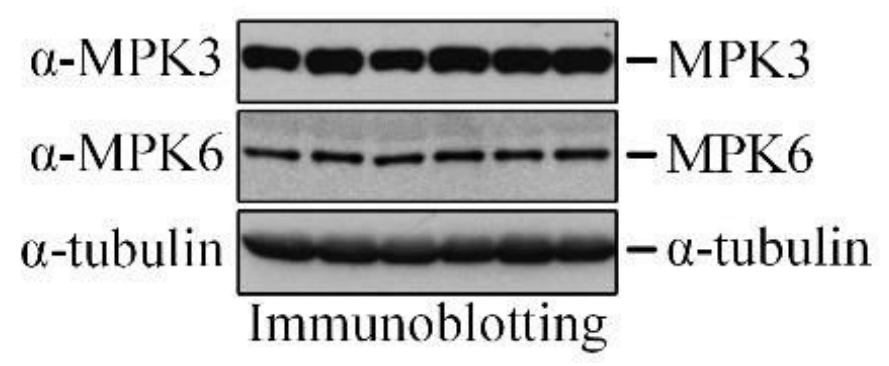

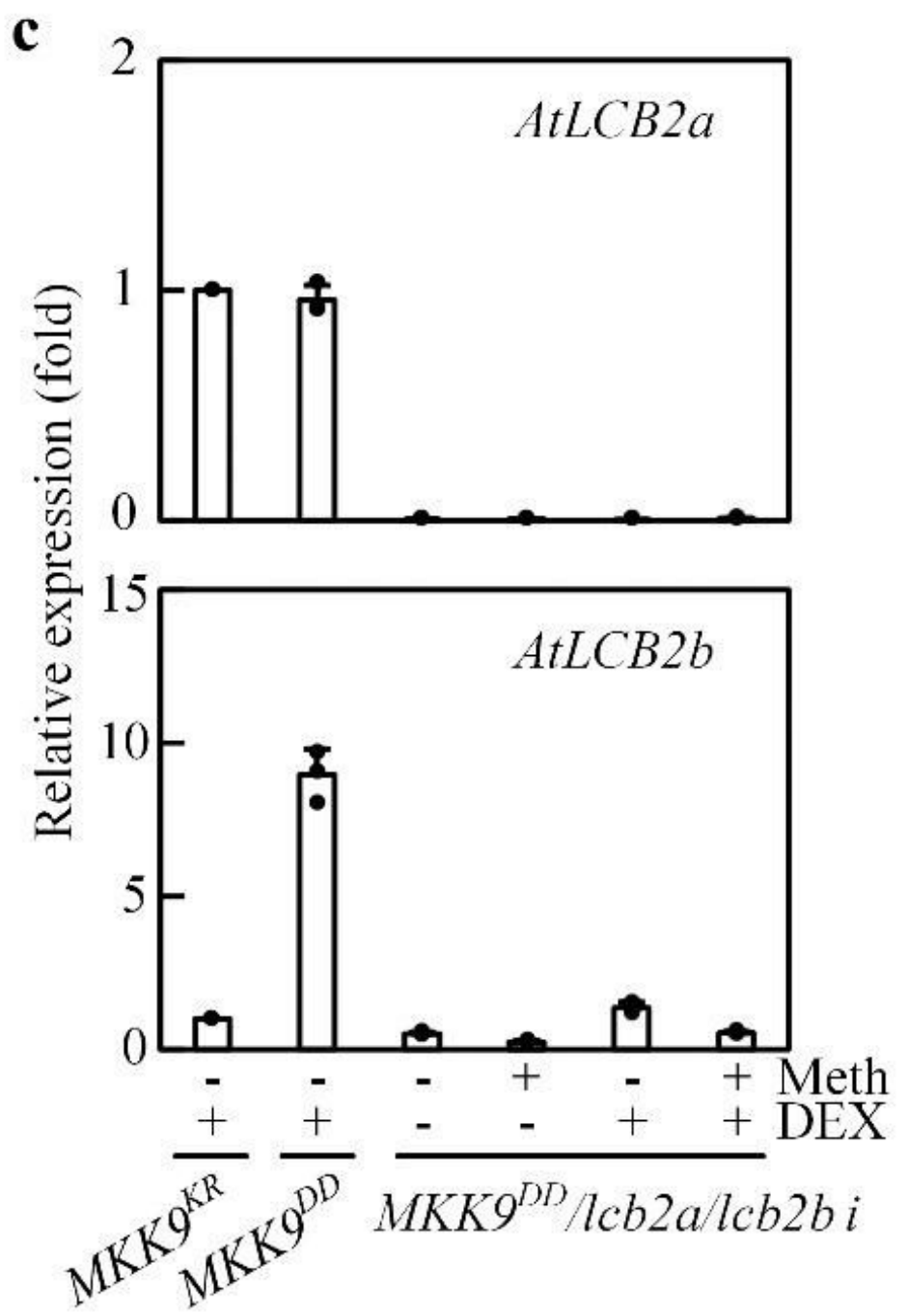

Figure 3

MKK9-MPK3/MPK6-induced LCB t18:0 is from its de novo biosynthesis. a, Free LCB t18:0 contents in MKK9KR, MKK9DD, and MKK9DD/lcb2a/lcb2b i seedlings with (+) or without (-) transgenes (MKK9KR, 
MKK9DD, and AtLCB2b RNAi) induction. $b$, In-gel kinase assay and immunoblotting detection of MPK3 and MPK6 in samples from seedlings treated as in (a); -tubulin was used as a loading control. c, Relative expression of AtLCB2a and AtLCB2b in the same groups of seedlings as in (a). Meth, methoxyfenozide (for induction of siRNA expression); DEX, dexamethasone (for induction of MKK9DD expression). Data represent the means $\pm S D$ of three biological replicates.



\section{Figure 4}

MKK9-MPK3/MPK6 phosphorylates AtLCB1 subunit in SPT. a, Yeast two-hybrid detection of interactions of MPK3 or MPK6 with various enzymes involved in LCB t18:0 biosynthesis and their regulators. $b, \mathrm{BiFC}$ analysis of the interaction of MPK3 or MPK6 with AtLCB1 using the tobacco leaf epidermal cell transient expression system. c, In vitro phosphorylation of the recombinant AtLCB1 variants by MKK9-activated MPK3 and MPK6. d, Phosphorylation of AtLCB1 in Col-0, mpk3, and mpk6 seedlings treated with Flg22 $(+)$ or not treated (-) and in MKK9DD, MKK9DD/mpk3, and MKK9DD/mpk6 seedlings grown with (+) or without (-) MKK9DD induction. Numbers in white show the ratios of phosphorylated to unphosphorylated AtLCB1. DEX, dethamethasone; CIAP, calf intestinal alkaline phosphatase. 
a

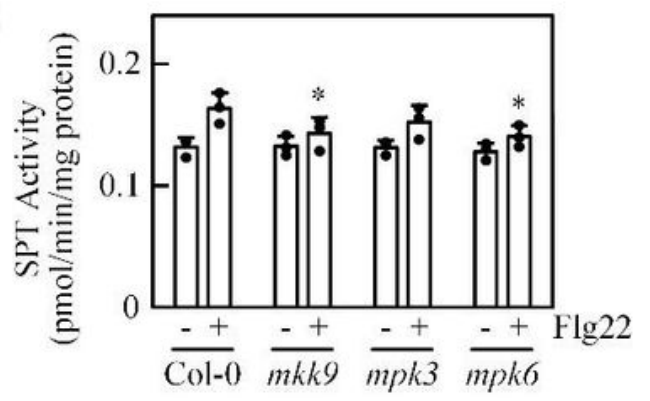

b

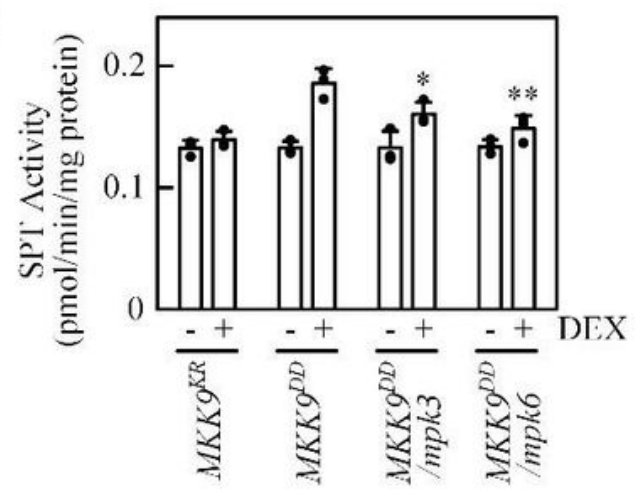

c
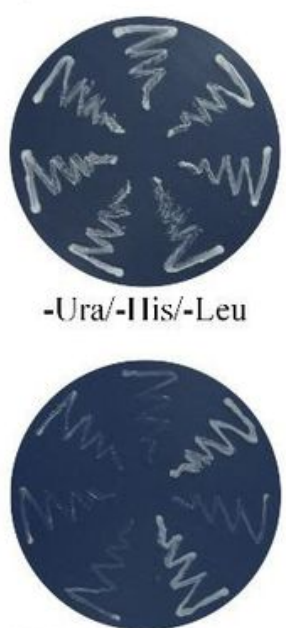

+5 -FOA/-His/-L eu



+5-FOA/-His/-Leu/ +PD98059

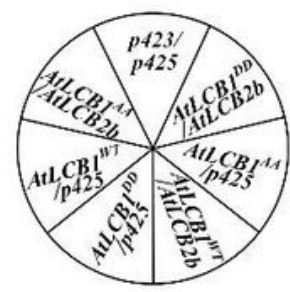

d

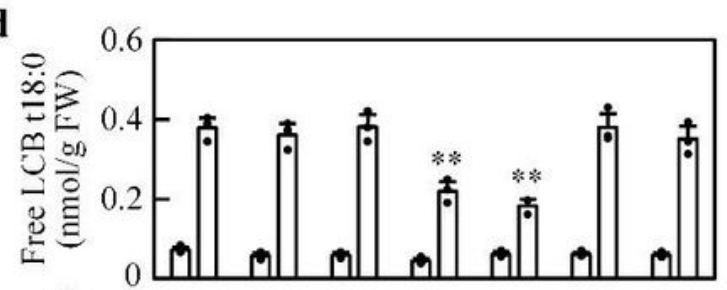

奉


e
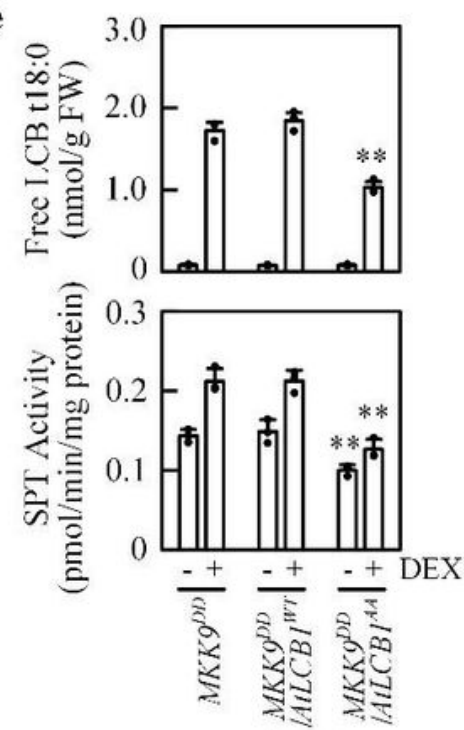

สิ

$\leq \leq \leq \leq$

$\frac{1}{-+} \frac{-1}{-+}$ DEX
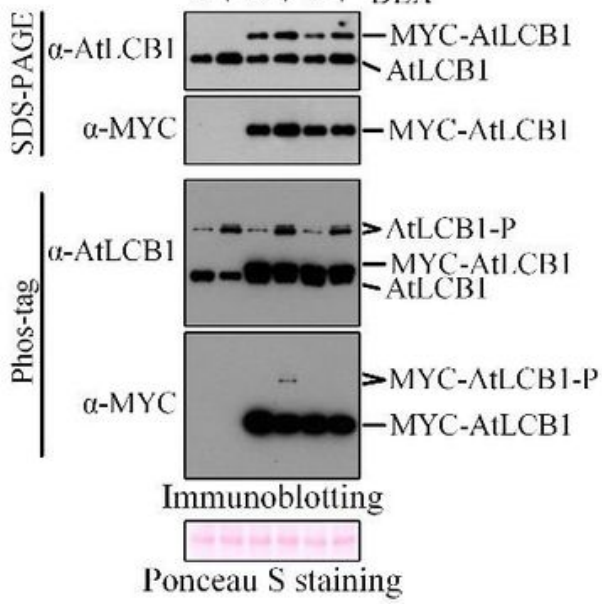

Figure 5

AtLCB1 phosphorylation stimulates SPT activity and free LCB t18:0 biosynthesis. a, SPT activities in Col0, mkk9, mpk3, and mpk6 seedlings treated with 9Flg22 (+) or not treated (-). Data represent the means \pm SD of three biological replicates. Asterisks indicate significant differences between Col-0 and mpk3 or mpk6 or mkk9 seedlings treated with Flg22 (two-tailed Student's t-test, ${ }^{\star} P<0.05$ ).b, SPT activities in MKK9KR, MKK9DD, MKK9DD/mpk3, and MKK9DD/mpk6 seedlings grown with (+) or without (-) 
transgenes induction. Data represent the means $\pm S D$ of three biological replicates. Asterisks indicate significant differences between Col-0 and mpk3 or mpk6 or mkk9 seedlings treated with Flg22 (two-tailed Student's t-test, $\left.{ }^{* *} \mathrm{P}<0.01\right)$.c, Yeast lcb1 mutant growth rescue by co-expression of AtLCB2b with AtLCB1 variant genes. $d$, SPT activities, free LCB t18:0 contents, and AtLCB1 phosphorylation in AtLCB1 variants transgenic seedlings treated with Flg22 (+) or not treated (-). e, SPT activities, free LCB t18:0 contents, and AtLCB1 phosphorylation in MKK9DD, MKK9DD/AtLCB1AA, and MKK9DD/AtLCB1WT seedlings grown with (+) or without (-) MKK9DD induction. ${ }^{*} \mathrm{P}<0.05,{ }^{* *} \mathrm{P}<0.01$, Student's t-test.

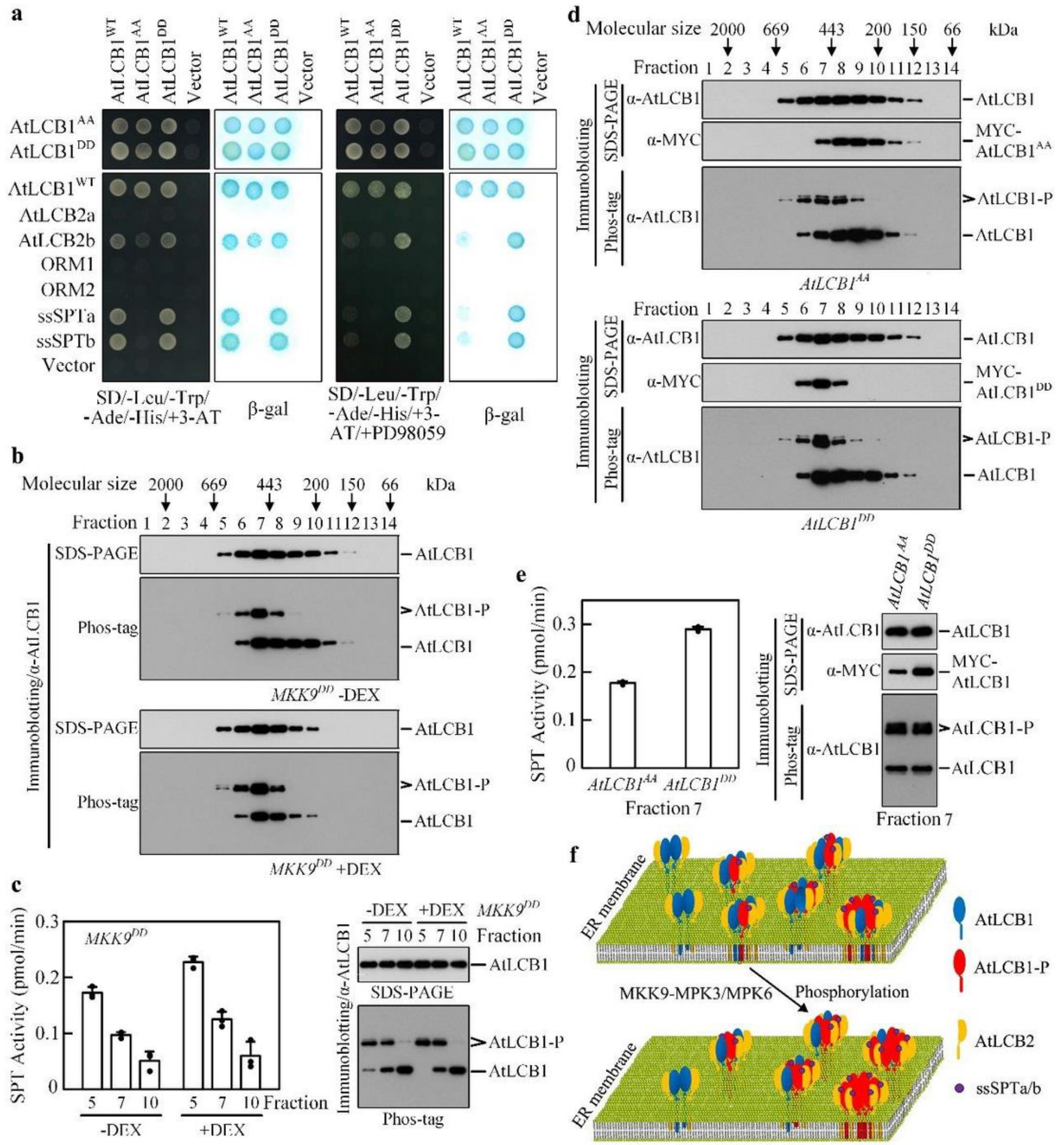




\section{Figure 6}

AtLCB1 phosphorylation stimulates higher oligomer and higher activity SPT formation. a, Yeast twohybrid detection of interactions between the AtLCB1 variants with each other and with the AtLCB2a, AtLCB2b, ORM1, ORM2, ssSPTa, and ssSPTb in the presence or absence of PD98059. b, Gel-filtration analysis of proteins from MKK9DD seedlings with or without MKK9DD induction. Fractions (1-14) were collected and analyzed for the presence of AtLCB1 by immunoblotting. c, SPT activities and AtLCB1 proteins in fractions 5,7 , and 10 from (b). Equal amount of AtLCB1 protein for each fraction was used for SPT activity assay. d, Gel filtration analysis of proteins from AtLCB1AA and AtLCB1DD seedlings. Fractions were collected and analyzed as described in (b). e, SPT activities and AtLCB1 proteins in fractions 5,7 , and 10 from (d). f, A working model for the regulation of SPT activity by AtLCB1 phosphorylation. 